\title{
Vitamin D receptor gene Fokl polymorphisms and tuberculosis susceptibility: a meta-analysis
}

\author{
Yan Cao, Xinjing Wang, Zhihong Cao, Xiaoxing Cheng
}

Key Laboratory of Tuberculosis Prevention and Treatment, and Beijing Key Laboratory of New Techniques for Tuberculosis Diagnosis and Treatment, Institute of Tuberculosis, $309^{\text {th }}$ Hospital, Beijing, China

Submitted: 24 January 2015

Accepted: 27 May 2015

Arch Med Sci 2016; 12, 5: 1118-1134

DOI: $10.5114 /$ aoms.2016.60092

Copyright $\odot 2016$ Termedia \& Banach

\section{Abstract}

Introduction: The association between Fokl polymorphism of vitamin D receptor (VDR) and tuberculosis (TB) susceptibility has been investigated previously; however, the results were inconsistent and conflicting. In the present study, a meta-analysis was performed to assess the relationship between VDR Fokl gene polymorphism and the risk of TB.

Material and methods: Databases including PubMed and Embase were searched for genetic association studies of Fokl polymorphism of vitamin D receptor (VDR) and TB. Data were extracted by two independent authors and the pooled odds ratio (OR) with 95\% confidence interval $(\mathrm{Cl})$ was calculated to assess the strength of the association between VDR Fokl gene polymorphism and TB risk. Meta-regression and subgroup analyses were performed to identify the source of heterogeneity.

Results: Thirty-four studies with a total of 5669 cases and 6525 controls were reviewed in the present meta-analysis. A statistically significant correlation was found between VDR FokI gene polymorphism and increased TB risk in two comparison models: the homozygote model ( $f f \mathrm{vs}$. FF: OR $=1.37$, $\left.95 \% \mathrm{Cl}: 1.17-1.60 ; P_{\text {heterogeneity }}=0.001\right)$ and the recessive model ( $\mathrm{ff} \mathrm{vs}$. Ff + FF: $\left.\mathrm{OR}=1.32,95 \% \mathrm{Cl}: 1.14-1.52 ; P_{\text {heterogeneity }}=0.006\right)$. Meta-regression found no source contributing to heterogeneity. However, sub-group analyses revealed that there was a statistically increased TB risk in the East and Southeast Asian population.

Conclusions: Synthesis of the available studies suggests that homozygosity for the FokI polymorphism of the VDR gene might be associated with an increased TB risk, especially in the East and Southeast Asian population. Additional well-designed, larger-scale epidemiological studies among different ethnicities are needed.

Key words: vitamin D receptor, Fokl polymorphisms, tuberculosis susceptibility.

\section{Introduction}

Tuberculosis (TB) is one of the most important infectious diseases, with an estimated 9.0 million new cases and 1.5 million deaths worldwide in 2013, and more than half were in the South-East Asia and Western Pacific Regions [1]. It is suggested that the susceptibility to disease after infection with Mycobacterium tuberculosis is influenced by many risk factors, such as malnutrition, HIV infection, and environmental and host genetic factors [2-5]. Host genetic factors implicated in human susceptibility

\author{
Corresponding author: \\ Xiaoxing Cheng MB, PhD \\ Division of Research \\ Institute of Tuberculosis \\ 309 Hospital \\ 17 Hei Shan Hu Road Haidian \\ Beijing 100091, China \\ Phone/fax: +86-10-51520496 \\ E-mail: xcheng2@139.com
}


to TB include NRAMP, HLA-DQB1, interleukin (IL) genes and the vitamin $D$ receptor (VDR) [6-8].

Vitamin $D$ deficiency seems to be involved in susceptibility to TB and severity of the disease [9], and 1,25-dihydroxyvitamin $D_{3}$, the activated form of vitamin $D$, is a potent immune modulator. Expression and nuclear activation of the VDR are essential for these activities of vitamin D. The VDR gene is located on the long arm of chromosome 12 , and several polymorphisms occur in the $5^{\prime}$ regulatory region, coding region and $3^{\prime}$ untranslated region (UTR) [10]. Among all the gene loci, the one most studied recently is Fokl [11-15], which can regulate the transcriptional activity of the gene [16]. Fokl polymorphism in combination with low serum vitamin $D_{3}$ may attenuate VDR functions, which in turn is strongly associated with TB [11]. Several studies have tried to investigate the role of FokI gene polymorphism on susceptibility to TB, but they have not reached a consensus. To date, two analyses on the Fokl polymorphism and TB risk across different ethnicities have been reported $[17,18]$, but they failed to identify a significant association of Fokl polymorphism in overall populations. In addition, more recent studies concerning the association between the polymorphism and TB risk in different populations have not included the two analyses [8, 12-15, 19-24]. Furthermore, several important factors which may bias the results were not clearly addressed, such as Hardy-Weinberg equilibrium (HWE). Thus, it is necessary to evaluate the true association of the VDR FokI gene polymorphism and the risk of TB. In the present study, we performed an updated meta-analysis to address these discrepancies and to explore the risk factors associated with TB.

\section{Material and methods}

\section{Literature search strategy}

We performed a literature search of the PubMed, Web of Science and Embase web databases with a combination of the key words "VDR" or "Vitamin D receptor"; "Fokl", "rs10735810"; "polymorphism" AND "Tuberculosis" up to January 2015. Furthermore, we evaluated potentially relevant genetic association studies by manual searching of references of relative articles and reviews. Search results were limited to human populations. No language restrictions were applied.

\section{Inclusion and exclusion criteria}

Published articles included in the current meta-analysis were selected according to the following criteria: (1) appraisal of the association between VDR Fokl gene polymorphism and TB risk, (2) case-control study design, (3) with clearly described and confirmed TB patients and TB- free controls, (4) containing available genotype frequency in cases and controls. The major reasons for study exclusion were data overlapping, case-only studies, reviews, repeated literature, and without genotype frequencies.

\section{Data extraction and quality assessment}

Data of each retrieved publication were independently abstracted in duplicate by two independent investigators with a standard procedure. Data extracted from the retrieved publications included the name of the first author, publication year, the country of origin, ethnicity, source of controls, number of cases and controls, study type, diagnosis method of cases, the selection of controls and genotype frequencies. The Hardy-Weinberg equilibrium (HWE) was examined by $\chi^{2}$ test $(p<$ 0.05 was considered as significant disequilibrium) based on Fokl genotyping distribution in controls.

\section{Statistical analysis}

Data from the meta-analysis were analyzed using STATA software (Version 12.1; Stata Corp, College Station, Texas, USA). The significance of the association for five comparison models - allele model ( $f$ vs. F), homozygote model (ff vs. FF), heterozygote model (Ff vs. FF), dominant model (ff + Ff vs. FF) and recessive model (ff vs. Ff + FF) - was evaluated for 34 studies separately. All associations were evaluated by calculating odds ratios (ORs) with the $95 \%$ confidence interval (Cl). The statistical heterogeneity between studies was checked using the $\chi^{2}$-based $\mathrm{Q}$ test and considered significant at $p<0.05$. When there was no significant heterogeneity, the fixed effects model (Mantel-Haenszel method) was used; otherwise, the random-effects model (the DerSimonian and Laird method) was used. Sensitivity analyses were performed to identify an individual study's effect on pooled results and test the reliability of results. Meta-regression analysis was performed to explore the source of potential heterogeneity. Stratification analyses were performed to further identify the possible source of heterogeneity among variables, such as ethnicity and sample size (studies with more than 500 participants were defined as "large", and studies with less than 500 participants were defined as "small"). Publication bias was assessed with both Egger's test and Begg's funnel plot, and the statistical significance was defined as $p<0.05$. All $p$ values were two-sided.

\section{Results}

\section{Characteristics of enrolled studies}

A flow chart of the study selection process is shown in Figure 1. According to the inclusion crite- 


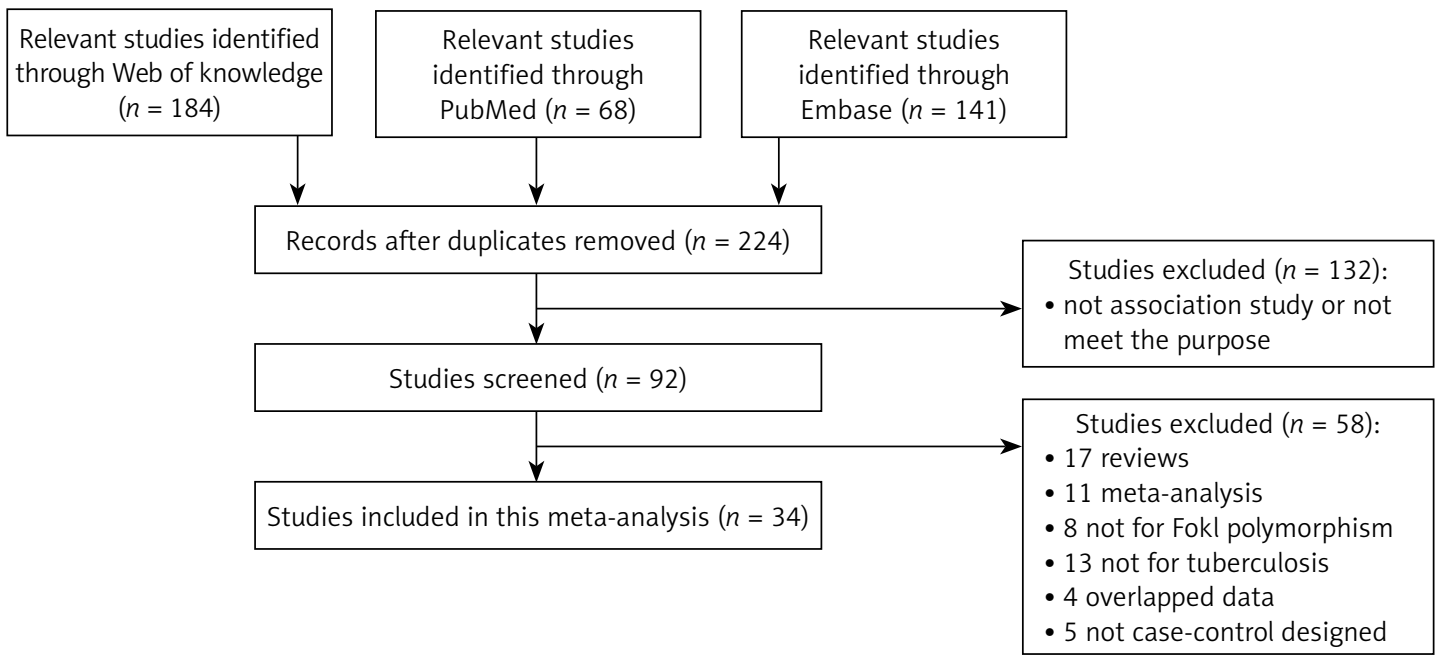

Figure 1. Flow diagram of search strategy and study selection process (TIF)

ria, 34 qualified case-control studies were selected in the final analysis after the literature search from the PubMed (Medline), Web of Science and Embase web databases [8, 10-15, 19-45]. Twenty-four studies were based on Asian populations [8, 10-13, 19-37], seven were based on African populations [14, 15, 38-42] and the remaining three were conducted in Europe and America [43-45]. The eligible studies contained 4 "large" studies $[19,29,40,42]$ and 30 "small" studies [8, 10-15, 21-28, 30-39]. Thirty studies were genotyped by restriction fragment length polymorphism (RFLP) analysis and five were conducted by other methods [19, 23, 38, 40,41]. The detailed characteristics of the enrolled studies are listed in Table I. A total of 5669 TB cases were obtained in the 34 studies, including 5126 (92.3\%) with pulmonary TB and 426 (7.7\%) with extra-pulmonary TB. The corresponding controls for the TB cases numbered 6525. Distribution of genotypes and HWE $p$-values in the controls are shown in Table II. Among the controls, the genotype distribution for 31 studies of the assessed polymorphisms was in HWE, except for 3 studies from India and Iran $[31,32,35]$.

\section{Sensitivity analyses and publication bias}

In the sensitivity analysis, the influence of each individual data set on the pooled OR was assessed by deleting one single study each time. The results showed that the corresponding pooled ORs were not materially varied, suggesting stability of this meta-analysis (data not shown). Begg's funnel plot and Egger's test were used to evaluate the publication bias of the selected studies for the meta-analysis (Figure 2). Begg's funnel plot seemed symmetrical in all genetic models. Furthermore, the statistical results from Egger's test supported the result of Begg's funnel plot indicat- ing that there was no publication bias among all genetic models $(p>0.05)$ (Table III).

\section{Meta-analysis results}

We pooled all 34 studies together for the assessment of the relationship between the VDR Fokl polymorphism and the risk of TB. The pooled ORs from overall studies indicated a significantly increased risk of TB in the homozygote model (ff vs. $\mathrm{FF}: \mathrm{OR}=1.37,95 \% \mathrm{Cl}: 1.17-1.60 ; P_{\text {h }}$ $=0.001$, Figure 3) and recessive model ( $f f$ Vs. $\mathrm{Ff}+\mathrm{FF}: \mathrm{OR}=1.32,95 \% \mathrm{Cl}: 1.14-1.52 ; P$ $=0.006$, Figure 4). However, no significant association was found in the allele model ( $f$ vs. F: $\mathrm{OR}=1.09,95 \% \mathrm{Cl}: 0.97-1.21 ; P_{\text {heterogeneity }}=0.000$, Figure 5) and in the dominant model ( $\mathrm{ff}+\mathrm{Ff}$ vs. FF: $\mathrm{OR}=1.08,95 \% \mathrm{Cl}: 0.99-1.17 ; P_{\text {heterogeneity }}=$ 0.000 , Figure 6). The heterozygote model (Ff vs. FF: $\mathrm{OR}=1.03,95 \% \mathrm{Cl}: 0.95-1.13 ; P_{\text {heterogeneity }}=$ 0.001 , Figure 7) failed to show any association with the risk of TB. The strength of the association between VDR FokI gene polymorphism and TB risk is shown in Table IV.

To account for the sources of heterogeneity, we performed meta-regression by publication years, ethnicity, sample size, genotyping methods, as well as source of controls and type of TB. However, no significant source was found to substantially contribute to heterogeneity (Table V).

To further investigate the heterogeneity, we performed subgroup analyses (Table IV). To evaluate the possible effect of the geographical differences on the variability of overall estimates, we classified the studies conducted in Asia into two groups: East and Southeast Asia (China, Indonesian and South Korean) and South and West Asia (India and Iran). As a result, the enrolled studies were divided into five subgroups including Africans, East and Southeast Asians, South and West 


\begin{tabular}{|c|c|c|c|c|c|c|c|c|c|c|}
\hline 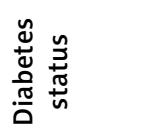 & 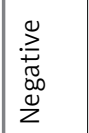 & 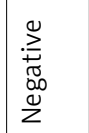 & 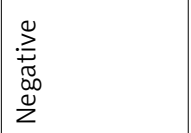 & 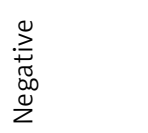 & 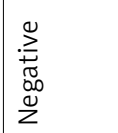 & 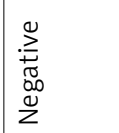 & 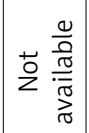 & 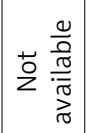 & 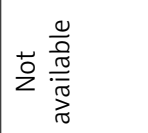 & 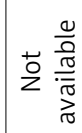 \\
\hline 离 & 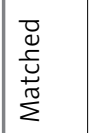 & 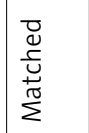 & 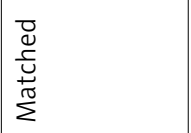 & 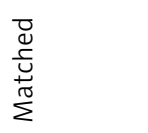 & 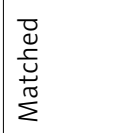 & 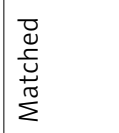 & 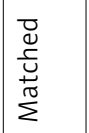 & 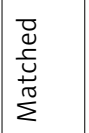 & 莡 & 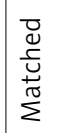 \\
\hline 主总 & 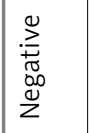 & 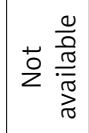 & 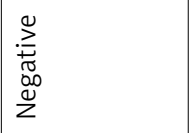 & 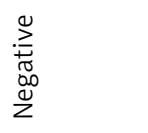 & 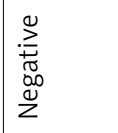 & 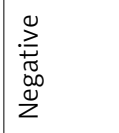 & 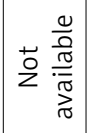 & 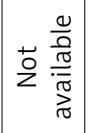 & 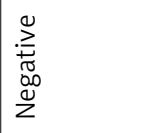 & 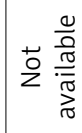 \\
\hline 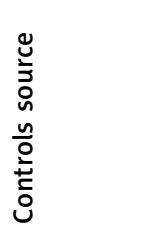 & 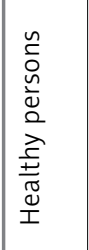 & 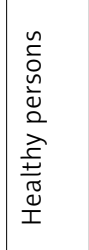 & 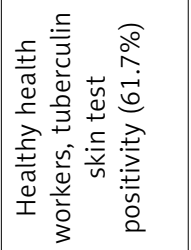 & 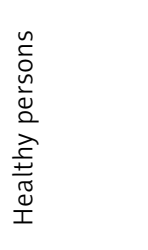 & 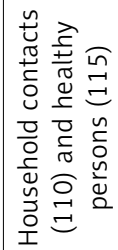 & 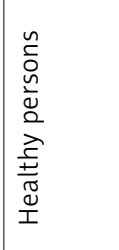 & 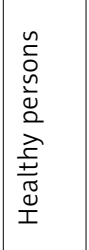 & 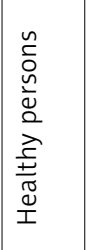 & 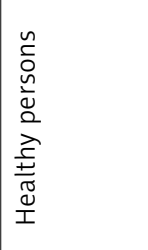 & 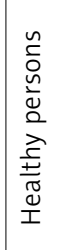 \\
\hline 它 & 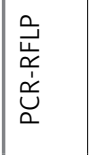 & 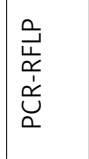 & 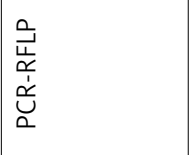 & 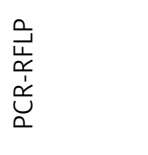 & 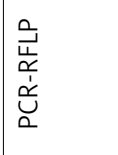 & 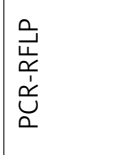 & 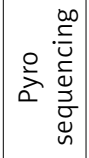 & 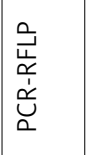 & 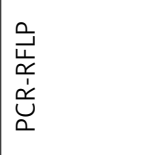 & 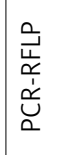 \\
\hline 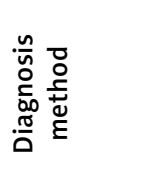 & 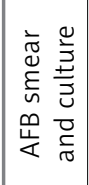 & 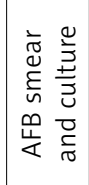 & 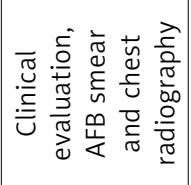 & 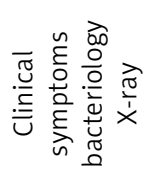 & 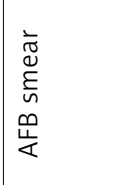 & 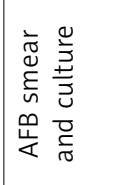 & 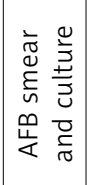 & 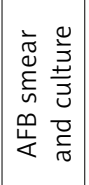 & 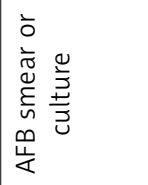 & 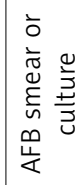 \\
\hline 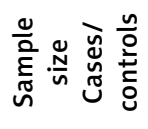 & 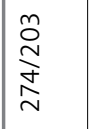 & $\stackrel{\stackrel{n}{\sim}}{\stackrel{q}{q}}$ & 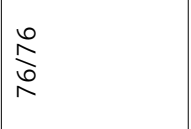 & 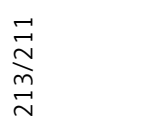 & 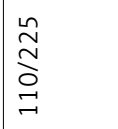 & 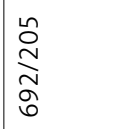 & 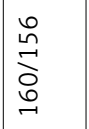 & 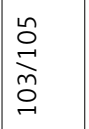 & 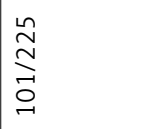 & \begin{tabular}{l}
$\hat{0}$ \\
0 \\
\multirow{2}{*}{} \\
$\dot{f}$
\end{tabular} \\
\hline 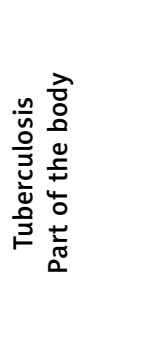 & 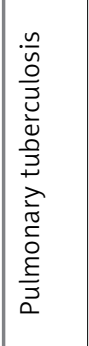 & 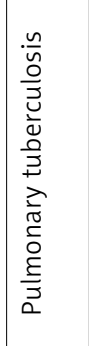 & 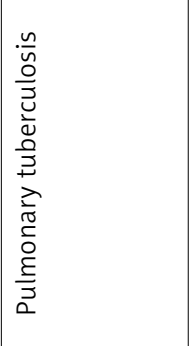 & 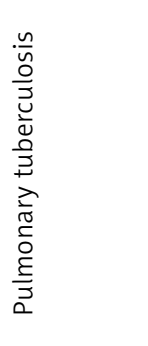 & 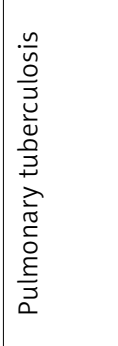 & 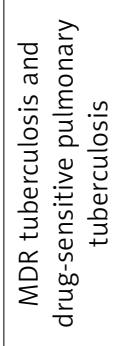 & 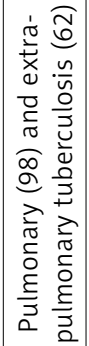 & 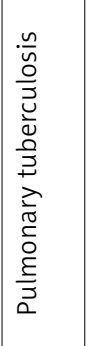 & 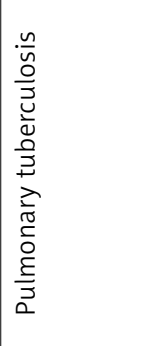 & 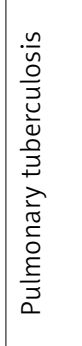 \\
\hline 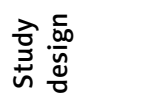 & 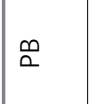 & 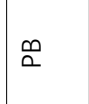 & 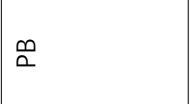 & 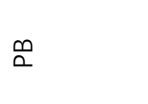 & 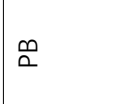 & 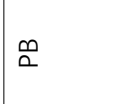 & $\ddot{\alpha}$ & $\stackrel{\infty}{2}$ & $\begin{array}{l}\infty \\
\frac{\infty}{x} \\
\frac{\rho}{I}\end{array}$ & 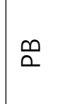 \\
\hline 童 & 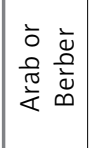 & 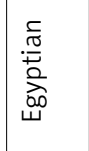 & 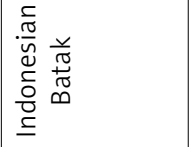 & 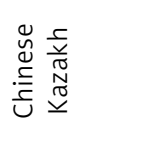 & 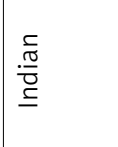 & 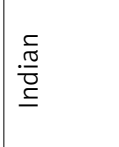 & 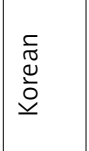 & 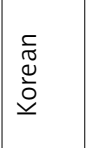 & 童 & 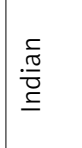 \\
\hline 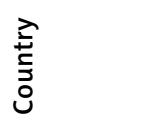 & 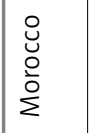 & 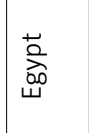 & 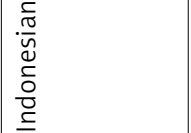 & 胥 & $\begin{array}{l}\text { 苪 } \\
\underline{\underline{\underline{I}}}\end{array}$ & 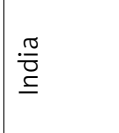 & 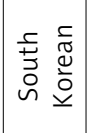 & 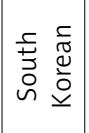 & $\begin{array}{l}\frac{\underline{\sigma}}{\overline{\underline{\theta}}} \\
\underline{\underline{y}}\end{array}$ & $\begin{array}{l}\stackrel{.}{\underline{\underline{\sigma}}} \\
\underline{\underline{\underline{I}}}\end{array}$ \\
\hline 蒙旁 & 妾 & 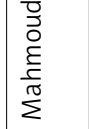 & 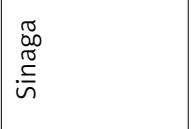 & $\xi_{3}^{5}$ & $\begin{array}{l}\text { 产 } \\
\text { 음 }\end{array}$ & 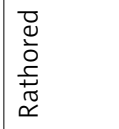 & 部 & 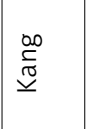 & $\begin{array}{l}\frac{c}{00} \\
\stackrel{\complement}{n}\end{array}$ & \begin{tabular}{|l}
$\frac{\pi}{\xi}$ \\
$\frac{\pi}{\omega}$ \\
$\tilde{\omega}$
\end{tabular} \\
\hline 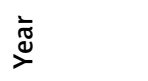 & $\stackrel{+}{\stackrel{\sim}{\sim}}$ & $\stackrel{\vec{D}}{\stackrel{\sim}{\sim}}$ & $\underset{\sim}{\stackrel{\sim}{\sim}}$ & $\stackrel{m}{\stackrel{n}{N}}$ & 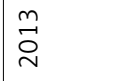 & $\underset{\sim}{\sim}$ & $\overrightarrow{\vec{D}}$ & $\vec{\sim}$ & $\vec{\sim}$ & $\overrightarrow{\vec{D}}$ \\
\hline
\end{tabular}




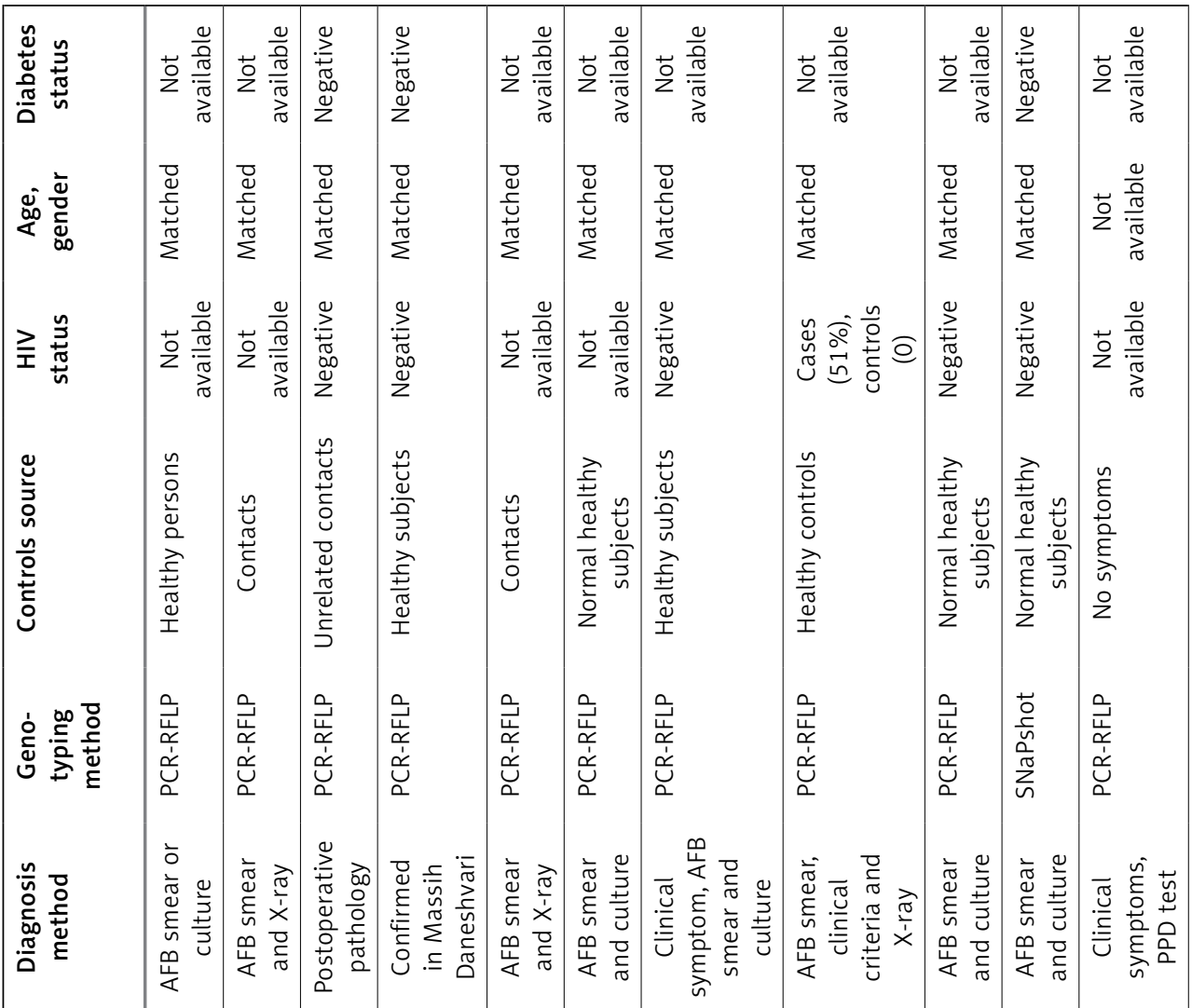

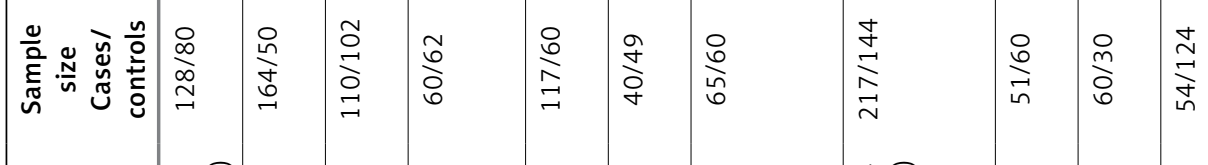

\begin{tabular}{|c|c|c|c|c|c|c|c|c|c|}
\hline 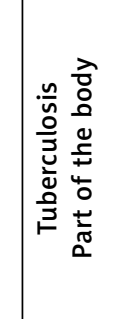 & 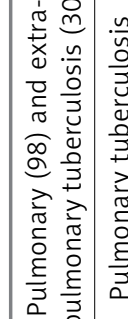 & 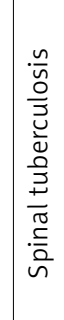 & 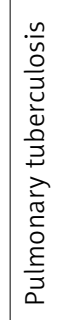 & 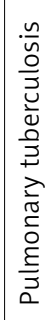 & 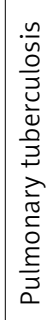 & 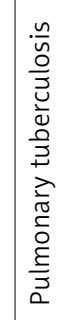 & 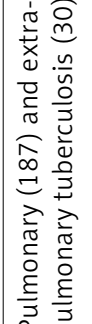 & 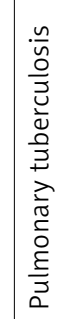 & 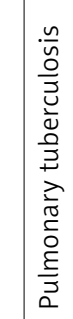 \\
\hline
\end{tabular}

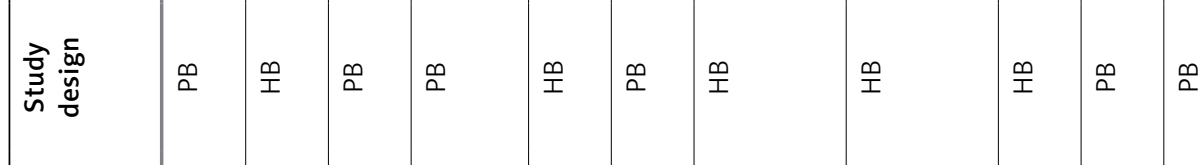

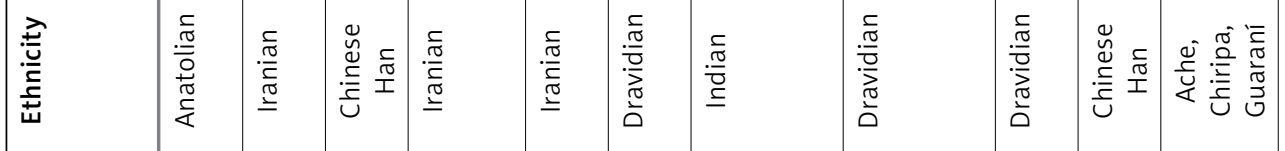

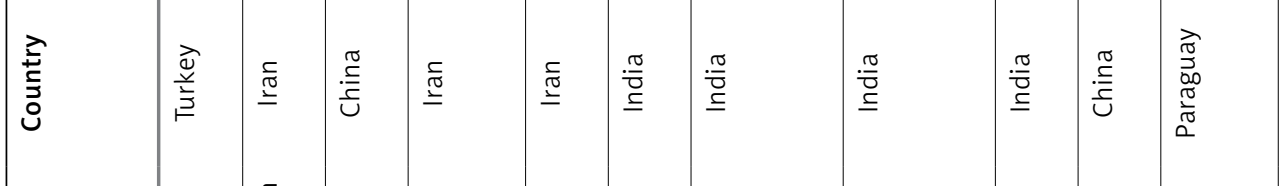

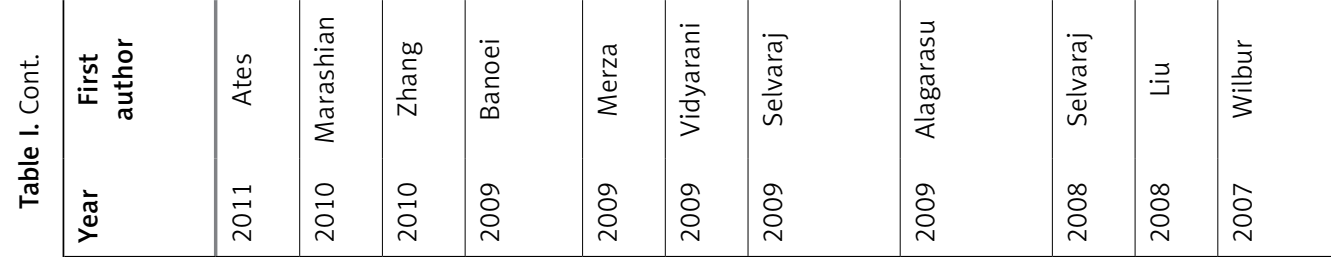




\begin{tabular}{|c|c|c|c|c|c|c|}
\hline 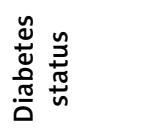 & $+\frac{\frac{0}{0}}{2} \underset{\substack{\frac{\pi}{\pi} \\
\frac{\pi}{\pi}}}{\frac{\pi}{\pi}}$ & 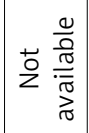 & 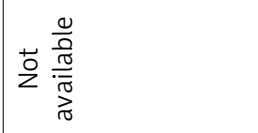 & 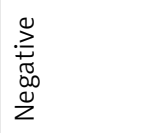 & $+\frac{\frac{0}{0}}{2} \underset{\frac{\pi}{\pi}}{\frac{\pi}{\pi}}$ & $+\frac{\frac{0}{0}}{2} \underset{\frac{\pi}{\pi}}{\frac{\pi}{\pi}}$ \\
\hline 离 & 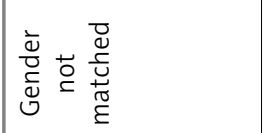 & $\frac{\frac{0}{0}}{\frac{0}{0}}$ & 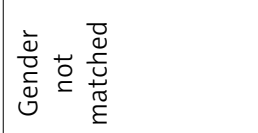 & 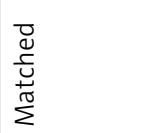 & 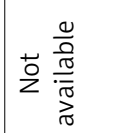 & 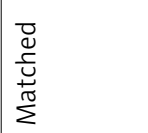 \\
\hline 》焉 & 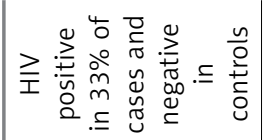 & 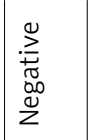 & 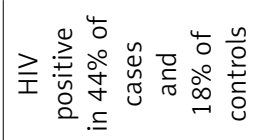 & 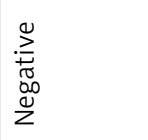 & 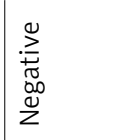 & 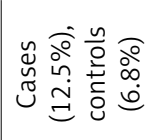 \\
\hline 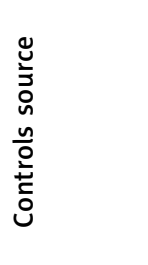 & 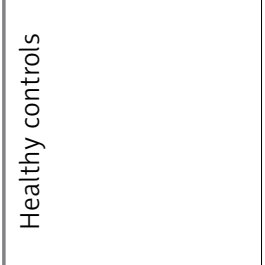 & 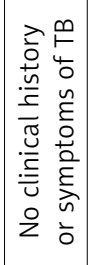 & 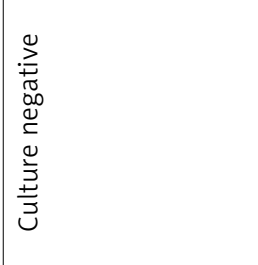 & 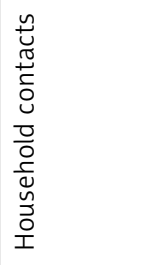 & 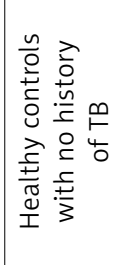 & 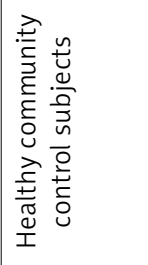 \\
\hline 定 & 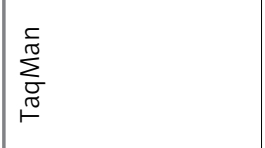 & 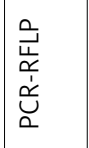 & $\begin{array}{l}\hat{\tilde{n}} \\
\hat{u} \\
\tilde{u} \\
\alpha\end{array}$ & 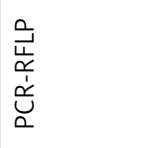 & 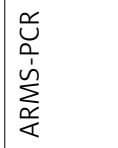 & 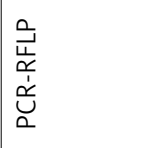 \\
\hline 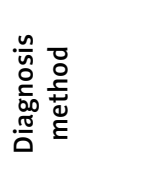 & 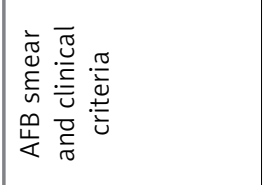 & 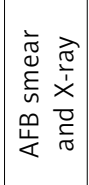 & $\frac{0}{3}$ & 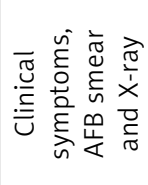 & 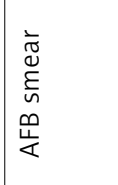 & 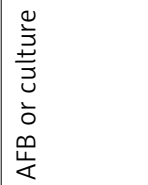 \\
\hline 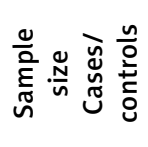 & 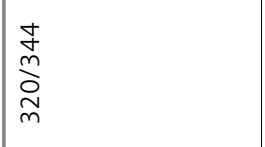 & 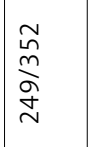 & 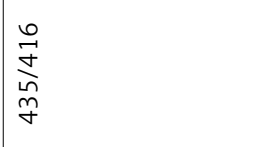 & $\underset{\substack{o \\
m}}{\stackrel{o}{d}}$ & $\begin{array}{l}\infty \\
\infty \\
\stackrel{\infty}{0} \\
ٍ\end{array}$ & $\begin{array}{l}\infty \\
\stackrel{1}{\hat{\sigma}} \\
\stackrel{\sigma}{\sigma}\end{array}$ \\
\hline 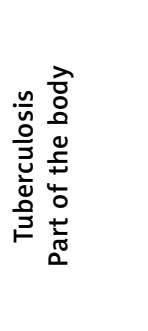 & 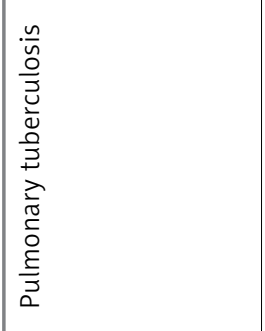 & 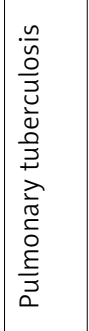 & 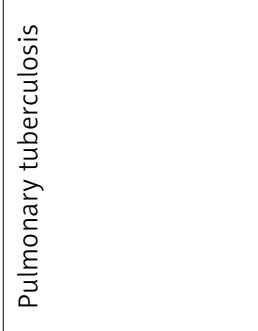 & 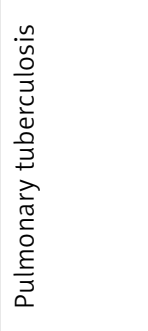 & 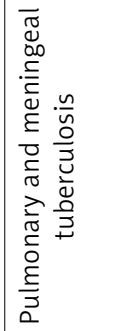 & 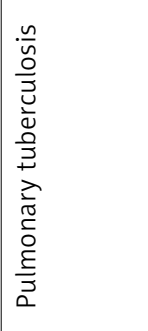 \\
\hline 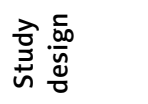 & 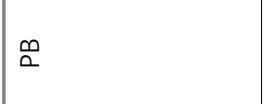 & $\dddot{\dddot{I}}$ & 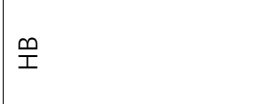 & 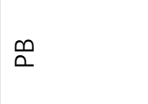 & $\dddot{I}$ & $\stackrel{\dddot{I}}{\underline{I}}$ \\
\hline 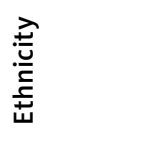 & 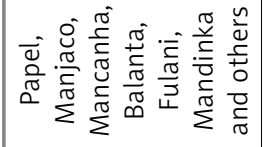 & 蛋 & 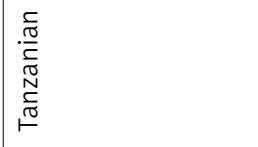 & 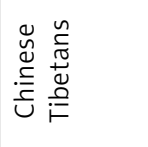 & $\begin{array}{l}\frac{\pi}{0} \\
\stackrel{\frac{\pi}{\pi}}{>}\end{array}$ & 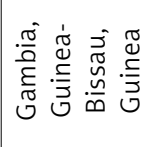 \\
\hline 产 & 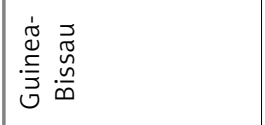 & 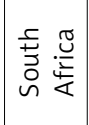 & 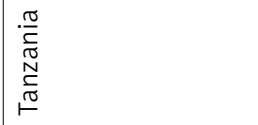 & 胥 & $\frac{\frac{\pi}{0}}{\frac{0}{\pi}}$ & 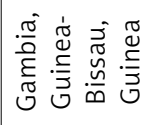 \\
\hline 点竞 & $\begin{array}{l}\bar{\Xi} \\
\bar{\circlearrowleft} \\
\overline{0}\end{array}$ & $\begin{array}{l}\frac{0}{0} \\
\infty \\
\infty\end{array}$ & $\begin{array}{l}\infty \\
\vdots \\
\vdots \\
0 \\
0 \\
\sim\end{array}$ & 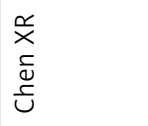 & 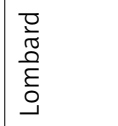 & 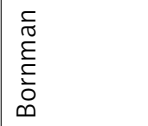 \\
\hline$\stackrel{\text { ঠ }}{\searrow}$ & চे & 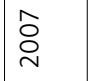 & 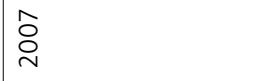 & ঃ & ¿্ণ & $\underset{\sim}{\stackrel{+}{0}}$ \\
\hline
\end{tabular}




\begin{tabular}{|c|c|c|c|c|c|c|c|}
\hline 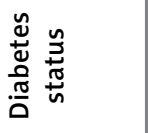 & 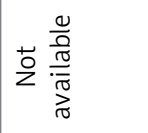 & 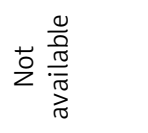 & 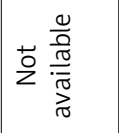 & 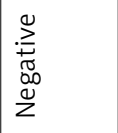 & 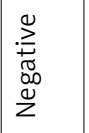 & 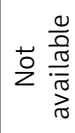 & $\frac{\frac{0}{0}}{2} \frac{\substack{\frac{0}{\pi} \\
\frac{\pi}{\pi}}}{\frac{\pi}{\pi}}$ \\
\hline 离 & 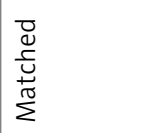 & 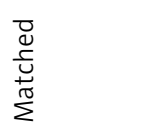 & 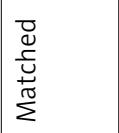 & 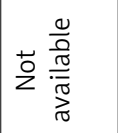 & 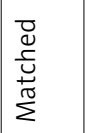 & 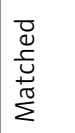 & 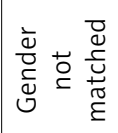 \\
\hline$\gtreqless \frac{n}{\underline{\underline{J}}}$ & 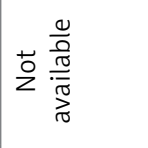 & 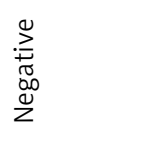 & 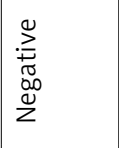 & 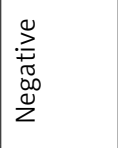 & 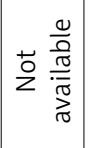 & 茓 & 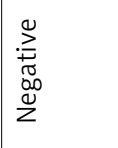 \\
\hline 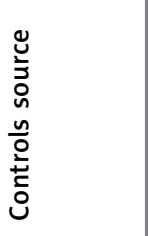 & 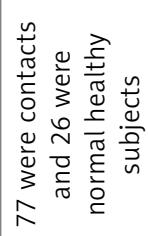 & 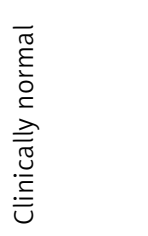 & 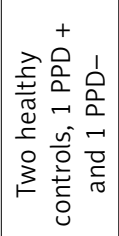 & 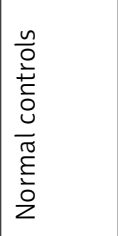 & 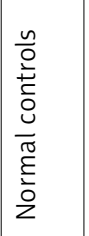 & 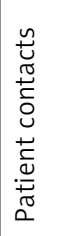 & 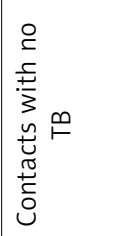 \\
\hline 它 & 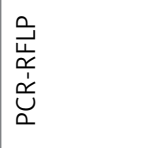 & 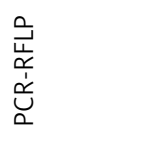 & 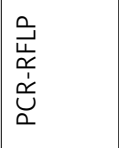 & 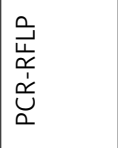 & 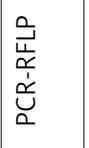 & 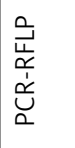 & 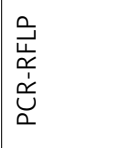 \\
\hline 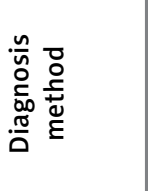 & 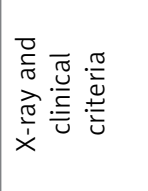 & 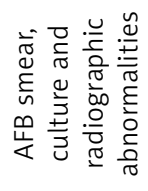 & 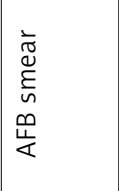 & 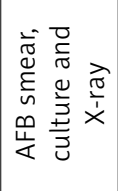 & 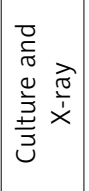 & $\stackrel{0}{\frac{0}{3}}$ & 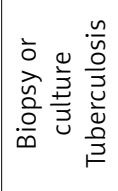 \\
\hline 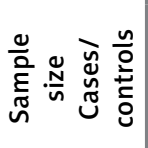 & 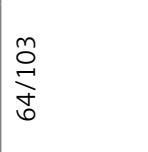 & 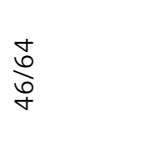 & 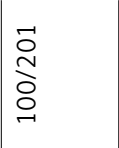 & 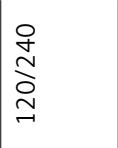 & 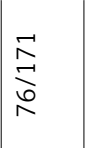 & 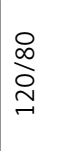 & $\underset{\sigma}{\stackrel{0}{\rightleftarrows}}$ \\
\hline 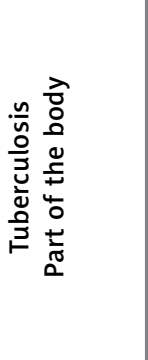 & 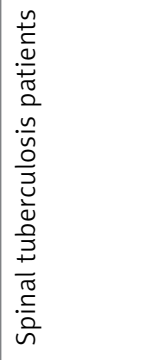 & 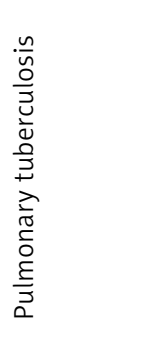 & 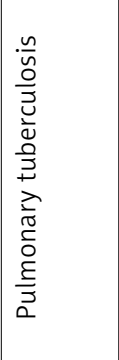 & 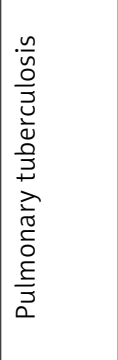 & 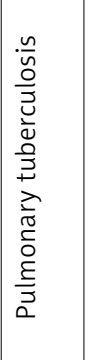 & 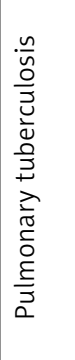 & 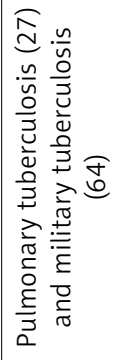 \\
\hline 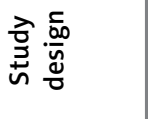 & 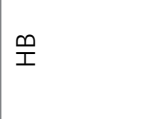 & $\stackrel{\cong}{\underline{I}}$ & 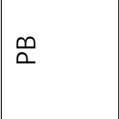 & $\unrhd$ & 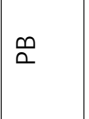 & 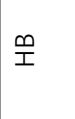 & $\stackrel{\oplus}{I}$ \\
\hline 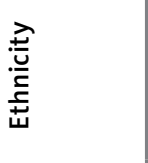 & 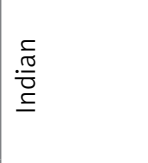 & 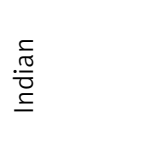 & 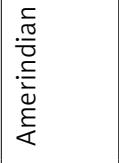 & 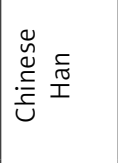 & 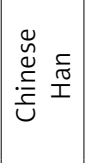 & 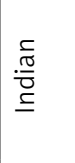 & 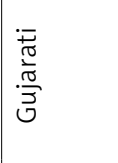 \\
\hline 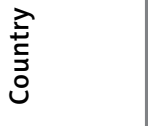 & $\frac{. \underline{\pi}}{\underline{\underline{\sigma}}}$ & $\begin{array}{l}\frac{\cdot \sqrt{0}}{\bar{\pi}} \\
\underline{\underline{I}}\end{array}$ & $\frac{2}{\frac{2}{0}}$ & 㢺 & : & $\begin{array}{l}\frac{\sigma \pi}{\pi} \\
\underline{\underline{I}}\end{array}$ & 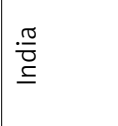 \\
\hline 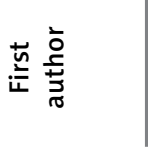 & 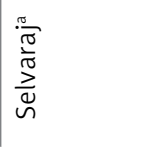 & $\mid$ & $\begin{array}{l}\stackrel{5}{+} \\
\stackrel{\circ}{\alpha}\end{array}$ & כבכ & כコ & 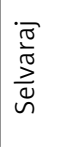 & 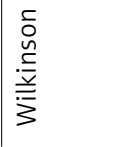 \\
\hline 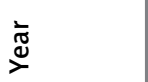 & $\underset{\sim}{+}$ & $\underset{\sim}{\stackrel{+}{~}}$ & $\underset{\sim}{\stackrel{D}{O}}$ & ণ্ণ & $\underset{\sim}{\stackrel{+}{O}}$ & 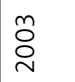 & 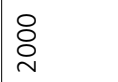 \\
\hline
\end{tabular}


Table II. Distribution of gene polymorphism of studies included in the meta-analysis

\begin{tabular}{|c|c|c|c|c|c|c|c|c|c|c|}
\hline \multirow[t]{3}{*}{ Year } & \multirow{3}{*}{$\begin{array}{l}\text { First } \\
\text { author }\end{array}$} & \multicolumn{5}{|c|}{ Case } & \multicolumn{3}{|c|}{ Control } & \multirow{3}{*}{$\begin{array}{c}\text { HWE } \\
P \text {-value }\end{array}$} \\
\hline & & \multicolumn{3}{|c|}{ Genotype } & \multirow{2}{*}{$\begin{array}{c}\begin{array}{c}\text { Minor } \\
\text { allele }\end{array} \\
\text { MAF }\end{array}$} & \multicolumn{3}{|c|}{ Genotype } & \multirow{2}{*}{$\begin{array}{c}\text { Minor } \\
\text { allele }\end{array}$} & \\
\hline & & $\mathrm{FF}$ & $\mathrm{Ff}$ & $\mathrm{ff}$ & & $\mathrm{FF}$ & Ff & $\mathrm{ff}$ & & \\
\hline 2014 & Arji & 151 & 103 & 20 & 0.26 & 109 & 82 & 12 & 0.26 & 0.5038 \\
\hline 2014 & Mahmoud & 12 & 20 & 8 & 0.45 & 10 & 10 & 5 & 0.4 & 0.404 \\
\hline 2014 & Sinaga & 27 & 42 & 7 & 0.37 & 30 & 34 & 12 & 0.38 & 0.6497 \\
\hline 2013 & Fang & 72 & 96 & 45 & 0.44 & 101 & 88 & 22 & 0.31 & 0.6642 \\
\hline 2013 & Joshi & 51 & 46 & 13 & 0.33 & 118 & 85 & 22 & 0.29 & 0.252 \\
\hline 2012 & Rathored & 319 & 298 & 75 & 0.32 & 118 & 80 & 7 & 0.23 & 0.1356 \\
\hline 2011 & Kim & 47 & 75 & 38 & 0.47 & 46 & 73 & 37 & 0.47 & 0.4463 \\
\hline 2011 & Kang & 30 & 58 & 15 & 0.43 & 41 & 43 & 21 & 0.40 & 0.1240 \\
\hline 2011 & Singh & 55 & 40 & 6 & 0.26 & 96 & 110 & 19 & 0.33 & 0.1069 \\
\hline 2011 & Sharma & 77 & 67 & 10 & 0.28 & 395 & 197 & 36 & 0.21 & 0.0880 \\
\hline 2011 & Ates & 58 & 60 & 10 & 0.31 & 35 & 37 & 8 & 0.33 & 0.6945 \\
\hline 2010 & Marashian & 97 & 57 & 10 & 0.23 & 15 & 30 & 5 & 0.40 & 0.0771 \\
\hline 2010 & Zhang & 16 & 43 & 51 & 0.66 & 26 & 47 & 29 & 0.51 & 0.4330 \\
\hline 2009 & Banoei & 30 & 21 & 9 & 0.33 & 29 & 27 & 6 & 0.31 & 0.9375 \\
\hline 2009 & Merza & 67 & 46 & 4 & 0.23 & 35 & 25 & 0 & 0.21 & 0.0415 \\
\hline 2009 & Vidyarani & 23 & 14 & 3 & 0.25 & 20 & 29 & 0 & 0.30 & 0.0033 \\
\hline 2009 & Selvaraj & 33 & 29 & 3 & 0.27 & 33 & 26 & 1 & 0.23 & 0.1019 \\
\hline 2009 & Alagarasu & 138 & 66 & 13 & 0.21 & 81 & 59 & 4 & 0.23 & 0.0766 \\
\hline 2008 & Selvaraj & 31 & 16 & 4 & 0.24 & 27 & 33 & 0 & 0.28 & 0.0033 \\
\hline 2008 & Liu & 16 & 25 & 19 & 0.53 & 11 & 17 & 2 & 0.35 & 0.1789 \\
\hline 2007 & Wilbur & 35 & 19 & 0 & 0.18 & 81 & 42 & 1 & 0.18 & 0.0740 \\
\hline 2007 & Olesen & 198 & 106 & 16 & 0.22 & 207 & 118 & 19 & 0.23 & 0.6862 \\
\hline 2007 & Babb & 132 & 104 & 13 & 0.26 & 203 & 129 & 20 & 0.24 & 0.9337 \\
\hline 2007 & Soborg & 288 & 128 & 19 & 0.19 & 267 & 128 & 21 & 0.20 & 0.2734 \\
\hline 2006 & Chen & 60 & 56 & 24 & 0.37 & 70 & 60 & 9 & 0.28 & 0.4144 \\
\hline 2006 & Lombard & 43 & 21 & 2 & 0.19 & 64 & 18 & 2 & 0.13 & 0.5917 \\
\hline 2004 & Bornman & 258 & 138 & 20 & 0.21 & 444 & 242 & 32 & 0.21 & 0.8932 \\
\hline 2004 & Selvaraja & 47 & 15 & 2 & 0.15 & 55 & 39 & 9 & 0.28 & 0.5834 \\
\hline 2004 & Selvaraj ${ }^{b}$ & 28 & 15 & 3 & 0.23 & 38 & 23 & 3 & 0.23 & 0.8388 \\
\hline 2004 & Roth & 9 & 32 & 59 & 0.75 & 14 & 78 & 109 & 0.74 & 0.9928 \\
\hline 2004 & Liu & 29 & 63 & 28 & 0.50 & 85 & 120 & 35 & 0.40 & 0.4821 \\
\hline 2004 & Liu W & 29 & 34 & 13 & 0.39 & 90 & 70 & 11 & 0.27 & 0.5930 \\
\hline 2003 & Selvaraj & 78 & 36 & 6 & 0.20 & 43 & 29 & 8 & 0.28 & 0.3551 \\
\hline 2000 & Wilkinson & 52 & 31 & 8 & 0.26 & 74 & 39 & 3 & 0.19 & 0.4178 \\
\hline
\end{tabular}

HWE - Hardy-Weinberg equilibrium, MAF - minor allele frequency, a,bthe different articles by the same author in the same year.

Asians, Americans and Europeans. As for ethnicities, an increased TB risk was found in the East and Southeast Asia population in five comparison models: allele model ( $\mathrm{f}$ vs. $\mathrm{F}$ : $\mathrm{OR}=1.42,95 \% \mathrm{Cl}$ : $\left.1.20-1.69 ; P_{\text {heterogeneity }}=0.055\right)$, homozygote model (ff vs. FF: OR = 1.98, 95\% Cl :1.53-2.56; $P$ $=0.012$ ), recessive model (ff vs. Ff + FF: OR = 1.64, 95\% Cl: 1.31-2.06; $\left.P_{\text {heterogeneity }}=0.003\right)$, heterozygote model (Ff vs. FF: OR $=1.37,95 \% \mathrm{Cl}: 1.13-$
1.65; $\left.P_{\text {heterogeneity }}=0.853\right)$ and dominant model $(\mathrm{ff}+\mathrm{Ff}$ vs. $\mathrm{FF}: \mathrm{OR}=1.52,95 \% \mathrm{Cl}: 1.27-1.82$; $\left.P_{\text {heterogeneity }}=0.695\right)$. In South and West Asians, however, no significant association was found in the heterozygote model (ff vs. Ff $+\mathrm{FF}: \mathrm{OR}=1.33$, 95\% Cl: 1.00-1.78; $P_{\text {heterogeneity }}=0.045$ ).

Further subgroup analyses were stratified by the source of the controls. Studies were divided into healthy persons-based and patient 
A

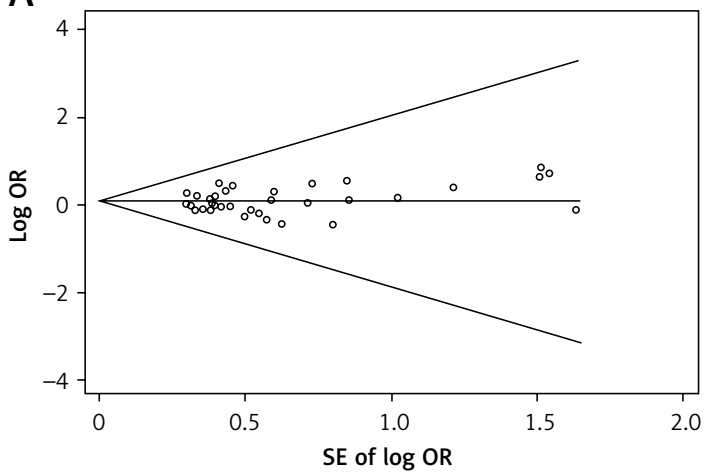

B

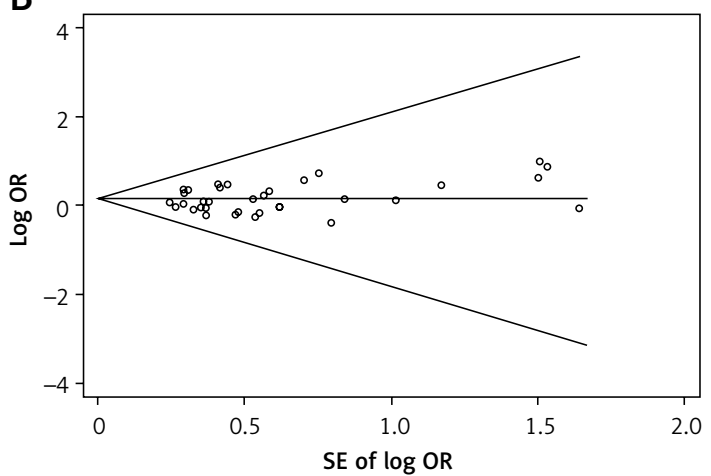

Figure 2. Funnel plot analysis to detect publication bias in 34 eligible studies. A - Funnel plot analysis of homozygote model (ff vs. FF). Egger's test $p=0.567$, Begg's test $p=0.423$; B - Funnel plot analysis of recessive model (ff vs. Ff $+\mathrm{FF}$ ). Egger's test $p=0.419$, Begg's test $p=0.343$; the circles represent the weight of individual study. log - logarithm, SE - standard error (TIF)

Table III. Statistics to test the publication bias and heterogeneity in the meta-analysis

\begin{tabular}{|lccccccc|}
\hline $\begin{array}{l}\text { Compa- } \\
\text { risons }\end{array}$ & $\begin{array}{c}\text { Begg's } \\
\text { regression } \\
\text { analysis }\end{array}$ & \multicolumn{2}{c}{ Egger's regression analysis } & & $\begin{array}{c}\text { Heterogeneity } \\
\text { analysis }\end{array}$ & $\begin{array}{c}\text { Model } \\
\text { used for } \\
\text { the } \\
\text { meta- } \\
\text { analysis }\end{array}$ \\
\hline f vs. F & 0.614 & $(-1.133)-0.404$ & 0.341 & 88.47 & 0.000 & 62.7 & Random \\
\hline ff vs. FF & 0.441 & $(-0.327)-0.574$ & 0.580 & 65.90 & 0.001 & 49.9 & Random \\
\hline Ff vs. FF & 0.313 & $(-0.949)-0.241$ & 0.234 & 66.35 & 0.001 & 50.3 & Random \\
\hline ff + Ff vs. FF & 0.459 & $(-0.918)-0.409$ & 0.440 & 79.44 & 0.000 & 58.5 & Random \\
\hline ff vs. Ff + FF & 0.495 & $(-0.327)-0.640$ & 0.514 & 57.01 & 0.006 & 42.1 & Random \\
\hline
\end{tabular}

contacts-based studies, and importantly the association in healthy persons-based studies was reinforced in the allele model ( $\mathrm{f}$ vs. $\mathrm{F}: \mathrm{OR}=1.13$, 95\% Cl: $\left.1.01-1.27 ; P_{\text {heterogeneity }}=0.001\right)$, the homozygote model (ff vs. FF: OR $=1.42,95 \% \mathrm{Cl}$ : $\left.1.18-1.70 ; P_{\text {heterogeneity }}=0.019\right)$ and the recessive model (ff vs. $\mathrm{Ff}+\mathrm{FF}$ : OR $=1.31,95 \% \mathrm{Cl}: 1.10-1.56$; $\left.P_{\text {heterogeneity }}=0.028\right)$, which conferred a significantly increased risk of TB, whereas this risk was reversed in patient contacts-based studies with no significance in each model (Table IV).

In addition, when categorized by the sample size with a cutoff of 500 individuals, 30 out of 34 studies had sample sizes less than 500 and conferred an increased risk of TB for two comparison models: the homozygote model (ff vs. FF: $\mathrm{OR}=1.38$, 95\% Cl: $\left.1.15-1.64 ; P_{\text {heterogeneity }}=0.002\right)$ and the recessive model (ff vs. $\mathrm{Ff}+\mathrm{FF}$ : $\mathrm{OR}=1.33,95 \% \mathrm{Cl}$ : $1.14-1.56 ; P_{\text {heterogeneity }}=0.012$ ). For the subgroup analysis by the genotyping methods, the homozygote model (ff vs. FF: OR $=1.47,95 \% \mathrm{Cl}$ : $\left.1.23-1.75 ; P_{\text {heterogeneity }}=0.001\right)$, recessive genetic model (ff vs. $\mathrm{Ff}+\mathrm{FF}: \mathrm{OR}=1.39,95 \% \mathrm{Cl}: 1.19$ 1.63; $\left.P_{\text {heterogeneity }}=0.010\right)$ and dominant model $(\mathrm{ff}+\mathrm{Ff}$ vs. FF: $\mathrm{OR}=1.10,95 \% \mathrm{Cl}: 1.00-1.20$; $\left.P_{\text {heterogeneity }}=0.000\right)$ remained statistically significant in PCR-RFLP studies (Table IV).

\section{Discussion}

Tuberculosis is one of the leading causes of morbidity and mortality, and the VDR gene might be important in modulating host susceptibility to TB because of the potential roles of VDR in the immune response to TB. However, many studies generated conflicting association data concerning the association between VDR Fokl gene polymorphism and the risk of TB.

Our present meta-analysis, based on 34 eligible studies until January 2015, provides evidence to propose a consistent effect of VDR FokI polymorphism. We found that the $f$ allele was associated with a significantly increased risk of TB in the homozygote model ( $\mathrm{ff}$ vs. FF) and the recessive model (ff vs. Ff + FF), especially in the East and Southeast Asian population. However, an insignificant association was found in South and West Asians, Africans, Americans and Europeans for all comparison models. To a certain extent, this finding could reflect the existence of racial differences, suggesting that this polymorphism might have a multifunctional role in the pathogenesis of TB or interact with other genetic and environmental factors. Previous studies including the WHO TB report suggested that the yellow race was more susceptible to TB than the black and white race [1]. 


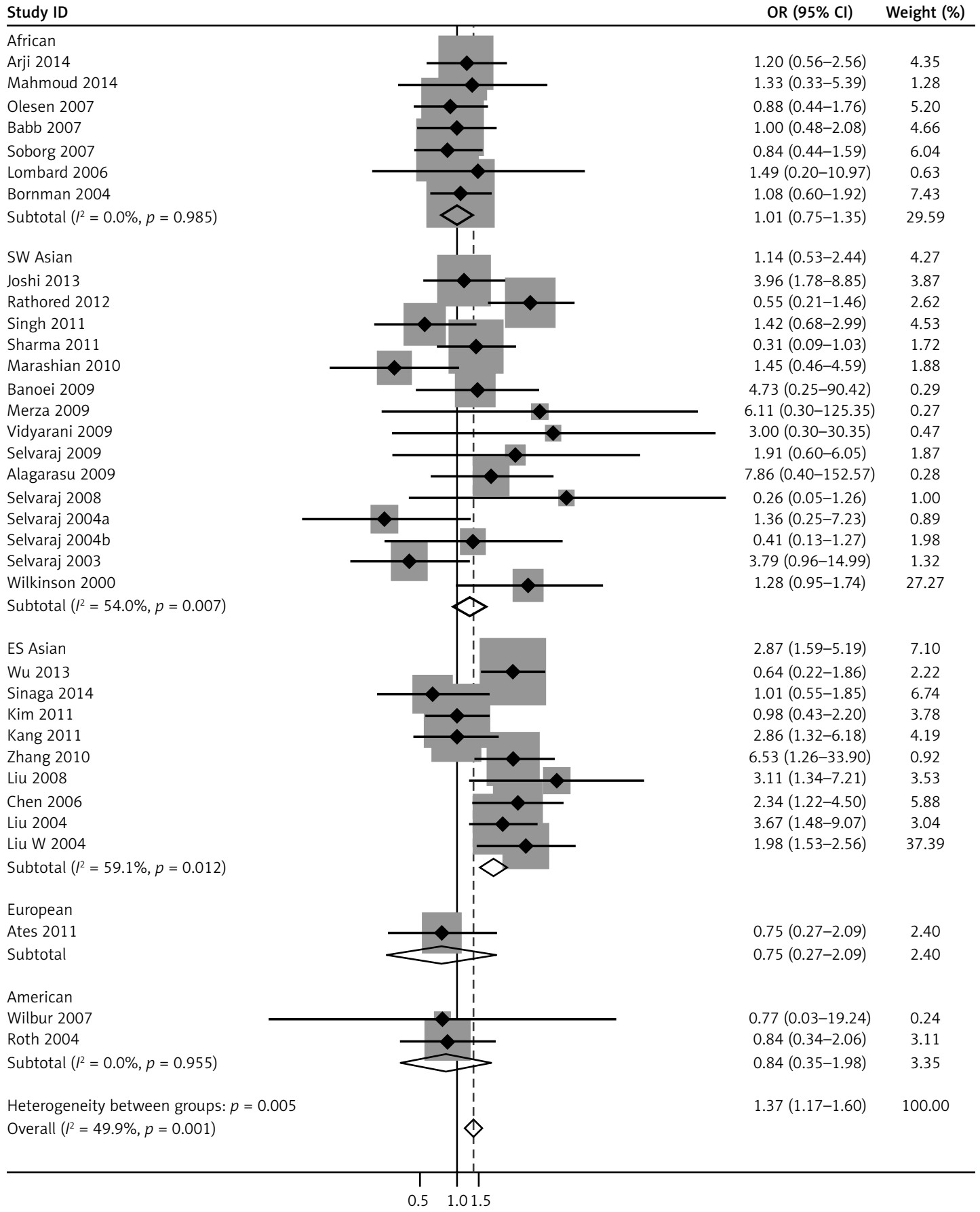

Figure 3. Forest plot of homozygote model for overall comparison (ff vs. FF) (TIF)

Additionally, it was reported that the $f$ allele frequency was higher in Asians than Africans [17]. Thus, the finding of this meta-analysis might be attributed to the racial differences.

There are some limitations to this systematic review. First, some individual information such as age, sex, HIV status and environmental factors could not be obtained, which makes the detailed sub-grouping analyses and interpretation of the results difficult. Second, considering that diabetes, hypertension and any other medical prob- lem may affect vitamin D level, the confounding effect should be taken into account. VDR Fokl polymorphisms have been suggested to be related to diabetes in Asians [46]. Diabetes status in the study population may therefore influence the association observed for VDR polymorphisms and TB incidence. Therefore, the stratification of diabetes status would further reveal the relationship between VDR gene SNPS and TB. However, diabetes status was not reported in two-thirds of the enrolled studies. Therefore, it was not possible to 


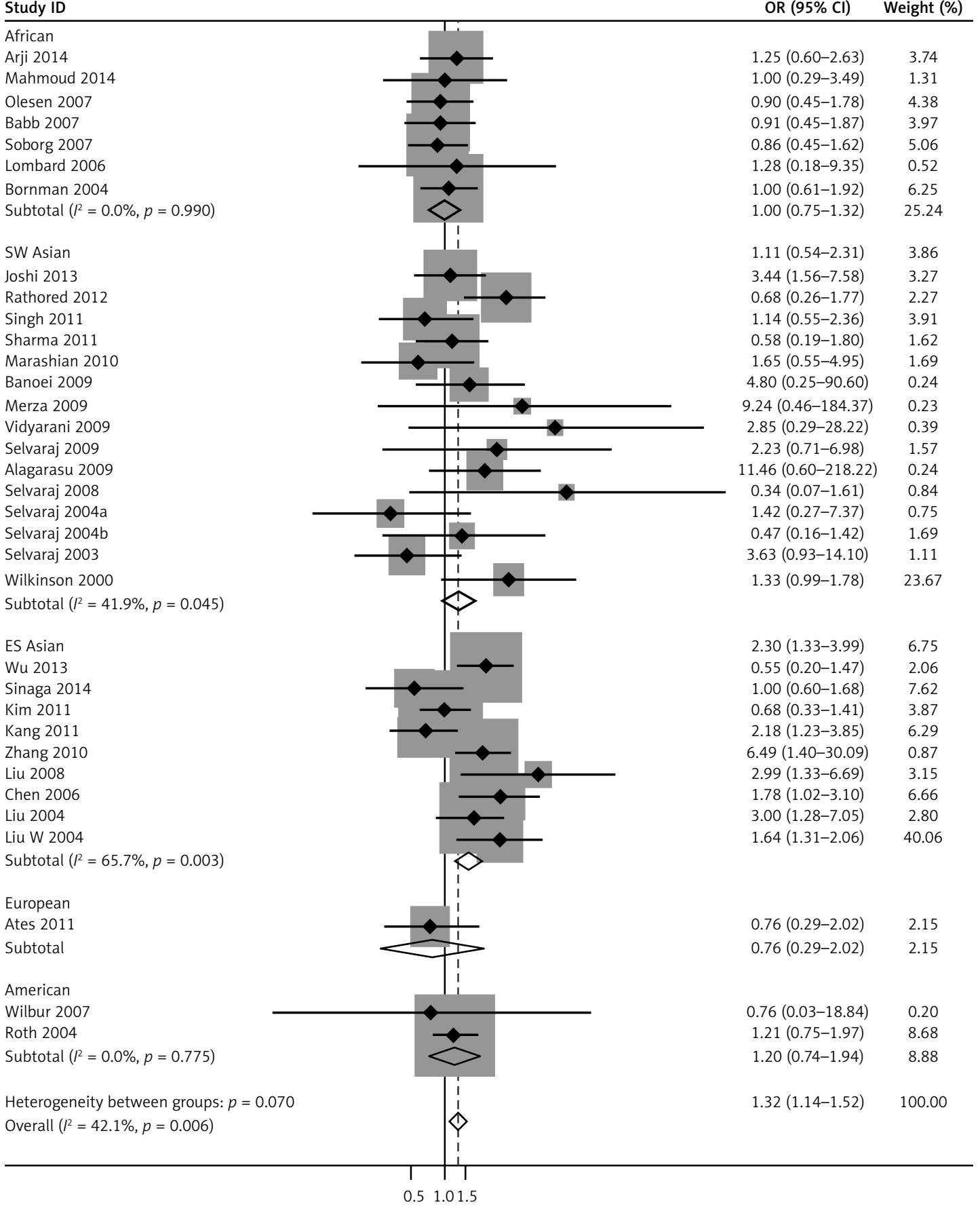

Figure 4. Forest plot of recessive model for overall comparison (ff vs. Ff + FF) (TIF)

apply stratification according to diabetes status. Third, the small sample sizes in some subgroup analyses may not comprehensively represent the population. More studies are needed to confirm the association of Fokl polymorphisms and TB risk, especially in different ethnic populations. Fourth, the different experimental designs and diagnostic standards make the analyses prone to bias. Fifth, included studies were restricted to those published in English or Chinese in our study, which might introduce potential bias into data analysis as well. Sixth, based on the data provided by the articles and our own calculations, significant deviations from HWE $(p<0.05)$ in controls were observed for three studies based on Asians [31, 32, 35]. Thus, their results should be interpreted with more caution. We therefore repeated the meta-analyses after exclusion of these studies. However, this exclusion did not materially affect the results (Table VI). Although genome-wide association studies (GWAS) are important for the discovery of genetic variations, we did not identify 


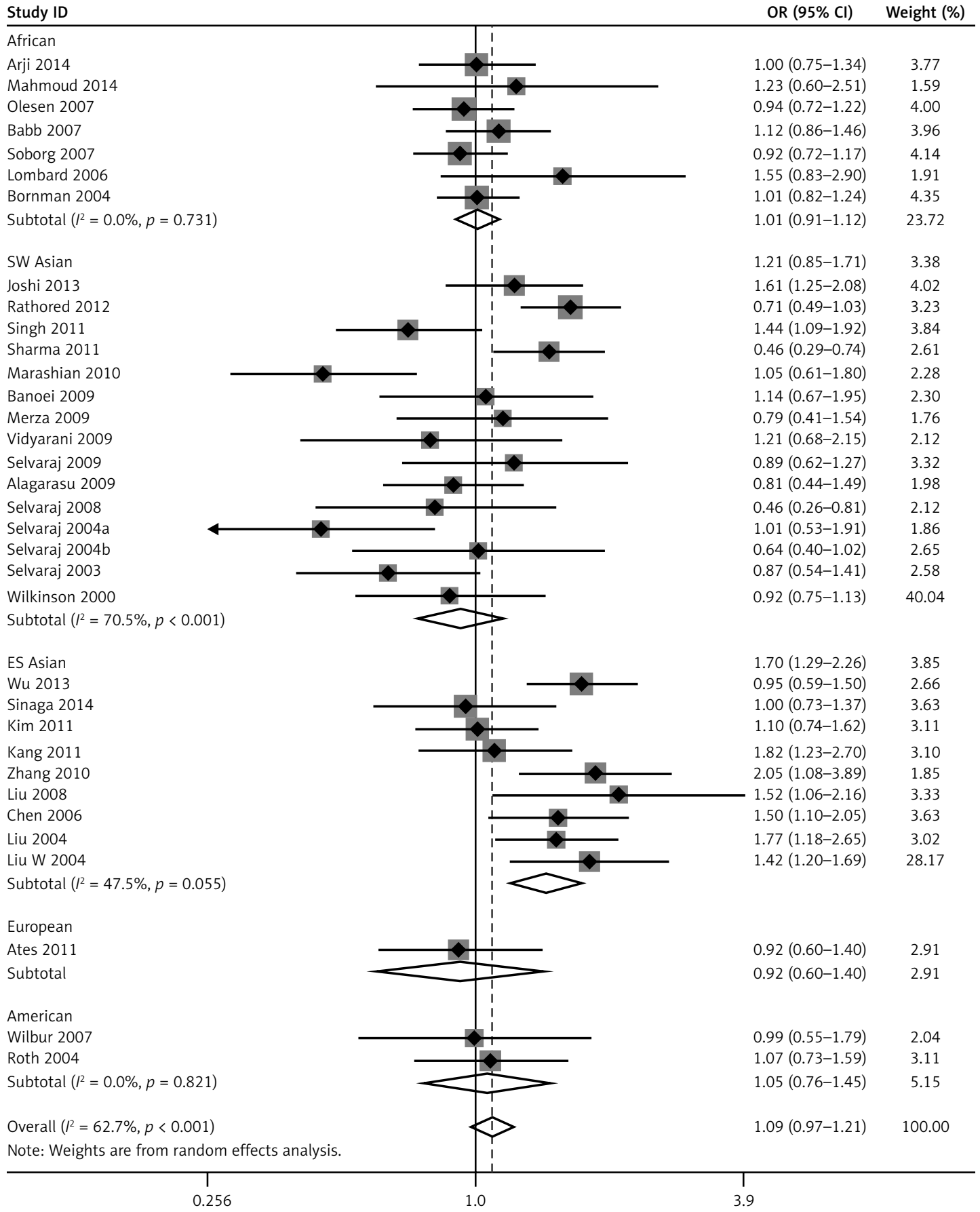

Figure 5. Forest plot of allele model for overall comparison ( $\mathrm{f}$ vs. F) (TIF)

any published GWAS on this subject. In conclusion, the results from this meta-analysis demonstrate that VDR FokI polymorphism is associated with increased TB risk, especially in East and Southeast Asians, which supports the hypothesis that VDR might play an important role in the host defense against TB. However, due to the moderate strength of the associations, their values to be used for risk prediction should be considered cautiously, and future large scale case-control studies are required to validate these findings.

\section{Acknowledgments}

Both authors - Yan Cao, Xinjing Wang - contributed equally to the work.

This study was supported by a key project of the $309^{\text {th }}$ Hospital to Y.C. (2014ZD-004). The funders had no role in study design, data collection and analysis, decision to publish, or preparation of the manuscript.

\section{Conflict of interest}

The authors declare no conflict of interest. 


\section{African}

Arji 2014

Mahmoud 2014

Olesen 2007

Babb 2007

Soborg 2007

Lombard 2006

Bornman 2004

Subtotal $\left(I^{2}=0.0 \%, p=0.525\right)$

\section{SW Asian}

Joshi 2013

Rathored 2012

Singh 2011

Sharma 2011

Marashian 2010

Banoei 2009

Merza 2009

Vidyarani 2009

Selvaraj 2009

Alagarasu 2009

Selvaraj 2008

Selvaraj 2004a

Selvaraj 2004b

Selvaraj 2003

Wilkinson 2000

Subtotal $\left(I^{2}=72.1 \%, p<0.001\right)$

ES Asian

Wu 2013

Sinaga 2014

Kim 2011

Kang 2011

Zhang 2010

Liu 2008

Chen 2006

Liu 2004

Liu W 2004

Subtotal $\left(I^{2}=0.0 \%, p=0.695\right)$

European

Ates 2011

Subtotal

American

Wilbur 2007

Roth 2004

Subtotal $\left(I^{2}=0.0 \%, p=0.592\right)$

Heterogeneity between groups: $p=0.001$ Overall $\left(R^{2}=58.5 \%, p<0.001\right)$

\begin{tabular}{r|r}
$i$ \\
\hline
\end{tabular}

$0.89(0.62-1.27) \quad 5.07$

$1.56(0.55-4.43) \quad 0.60$

$0.93(0.68-1.27) \quad 6.78$

$1.21(0.87-1.67) \quad 6.20$

$0.91(0.69-1.21) \quad 8.30$

$1.71(0.84-3.49) \quad 1.30$

$0.99(0.77-1.27) \quad 10.68$

$1.01(0.88-1.15) \quad 38.94$

$1.06(0.67-1.69) \quad 3.07$

$1.59(1.16-2.17) \quad 6.67$

$0.62(0.39-1.00) \quad 2.96$

$1.70(0.19-2.42) \quad 5.25$

$0.30(0.15-0.58) \quad 1.43$

$0.88(0.43-1.79) \quad 1.31$

$1.04(0.56-1.96) \quad 1.66$

$0.51(0.22-1.19) \quad 0.92$

$1.19(0.59-2.40) \quad 1.34$

$0.74(0.48-1.13) \quad 3.58$

$0.53(0.25-1.13) \quad 1.15$

$0.41(0.21-0.82) \quad 1.44$

$0.94(0.43-2.04) \quad 1.10$

$0.63(0.35-1.12) \quad 1.98$

$1.32(0.75-2.32) \quad 2.09$

$0.97(0.85-1.11) \quad 35.96$

$1.80(1.22-2.66) \quad 4.31$

$1.18(0.62-2.25) \quad 1.59$

$1.01(0.62-1.63) \quad 2.82$

$1.56(0.87-2.78) \quad 1.98$

$2.01(1.01-4.02) \quad 1.38$

$1.59(0.62-4.07) \quad 0.75$

$1.35(0.84-2.17) \quad 2.97$

$1.72(1.05-2.82) \quad 2.70$

$1.80(1.04-3.13) \quad 2.17$

$1.52(1.27-1.82) \quad 20.67$

$0.94(0.53-1.65) \quad 2.09$

$0.94(0.53-1.65) \quad 2.09$

$1.02(0.52-2.00) \quad 1.47$

$0.76(0.32-1.81) \quad 0.87$

$0.91(0.54-1.56) \quad 2.34$

$1.08(0.99-1.17) \quad 100.00$ 
Study ID

\section{African}

Arji 2014

Mahmoud 2014

Olesen 2007

Babb 2007

Soborg 2007

Lombard 2006

Bornman 2004

Subtotal $\left(R^{2}=0.0 \%, p=0.533\right)$

SW Asian

Joshi 2013

Rathored 2012

Singh 2011

Sharma 2011

Marashian 2010

Banoei 2009

Merza 2009

Vidyarani 2009

Selvaraj 2009

Alagarasu 2009

Selvaraj 2008

Selvaraj 2004a

Selvaraj 2004b

Selvaraj 2003

Wilkinson 2000

Subtotal $\left(I^{2}=68.8 \%, p<0.001\right)$

ES Asian

Wu 2013

Sinaga 2014

Kim 2011

Kang 2011

Zhang 2010

Liu 2008

Chen 2006

Liu 2004

Liu W 2004

Subtotal $\left(I^{2}=0.0 \%, p=0.853\right)$

European

Ates 2011

Subtotal

American

Wilbur 2007

Roth 2004

Subtotal $\left({ }^{2}=0.0 \%, p=0.399\right)$

Heterogeneity between groups: $p=0.021$

Overall $\left(R^{2}=50.3 \%, p=0.001\right)$

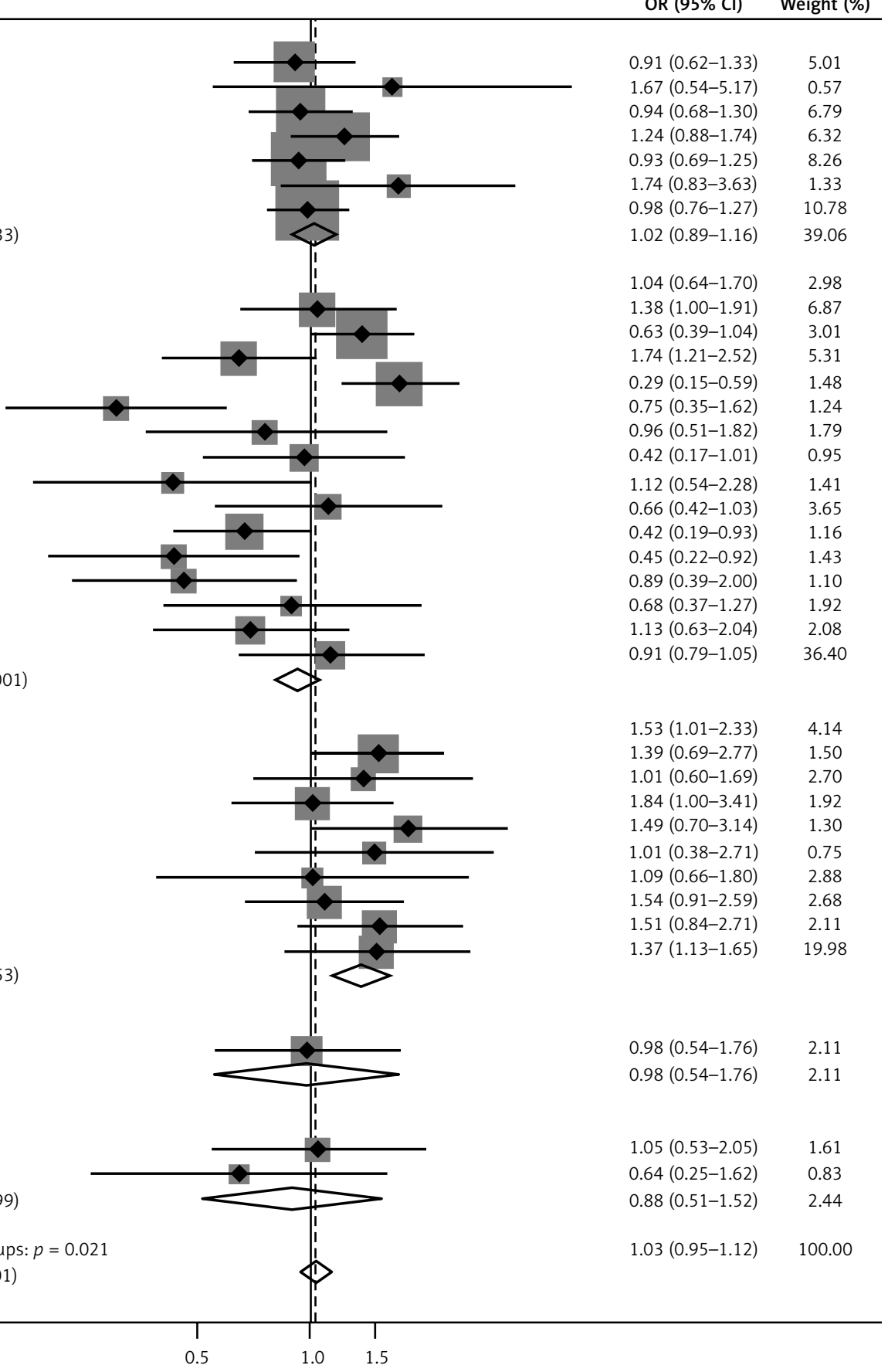

Figure 7. Forest plot of heterozygote model for overall comparison (Ff vs. FF) (TIF)
OR $(95 \% \mathrm{Cl}) \quad$ Weight $(\%)$

$91(0.62-1.33) \quad 5.01$

$1.67(0.54-5.17) \quad 0.57$

$9.94(0.68-1.30)$

$\begin{array}{ll}1.24(0.88-1.74) & 6.32 \\ 0.93(0.69-1.25) & 8.26\end{array}$

$1.74(0.83-3.63) \quad 1.33$

$0.98(0.76-1.27) \quad 10.78$

$1.74(1.21-2.52) \quad 5.31$

$0.29(0.15-0.59) \quad 1.48$

$0.75(0.35-1.62) \quad 1.24$

(0.51-1.82) 1.79

$0.42(0.19-0.93)$

$0.45(0.22-0.92) \quad 1.43$

$0.89(0.39-2.00) \quad 1.10$

$1.13(0.63-2.04)-2.08$

$1.53(1.01-2.33) \quad 4.14$

$139(0.69-2.77)-1.50$

$1.01(0.38-2.71)$

$1.09(0.66-1.80)-2.88$

$1.54(0.91-2.59) \quad 2.68$

$1.51(0.84-2.71) \quad 2.11$

$1.37(1.13-1.65) \quad 19.98$

$0.98(0.54-1.76) \quad 2.11$

$0.98(0.54-1.76) \quad 2.11$

$1.05(0.53-2.05) \quad 1.61$

$0.64(0.25-1.62) \quad 0.83$

$1.03(0.95-1.12) \quad 100.00$ 


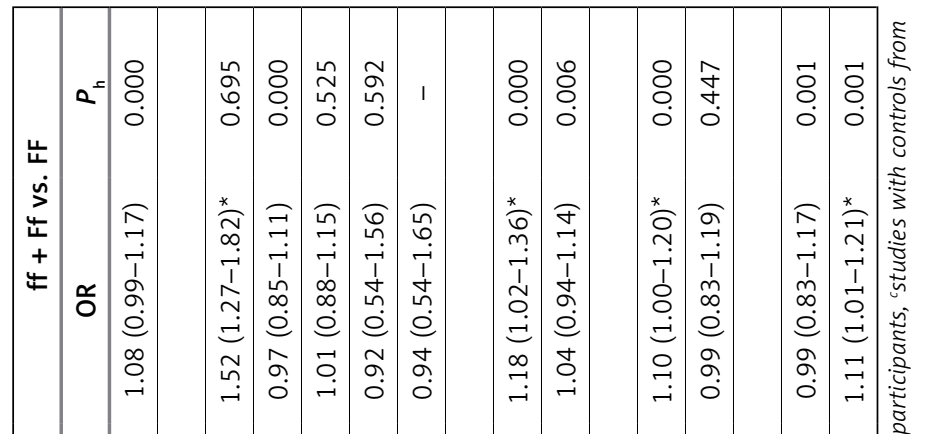

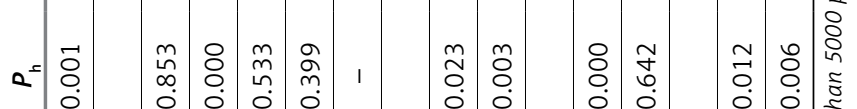

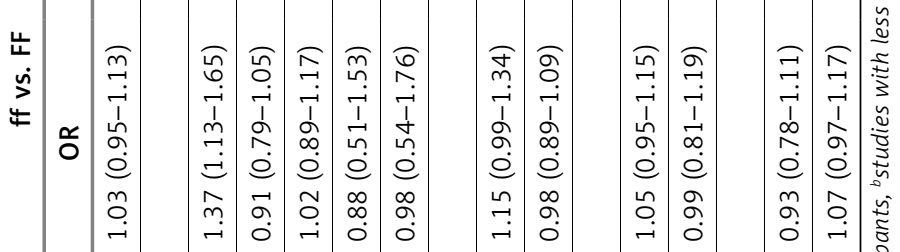

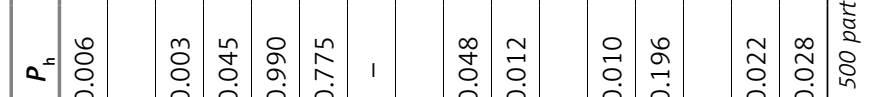

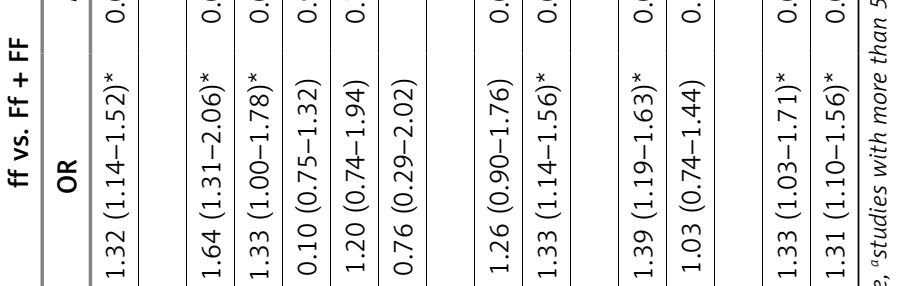

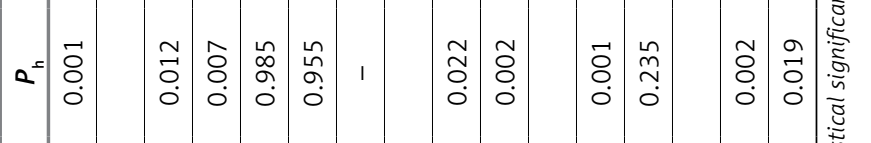

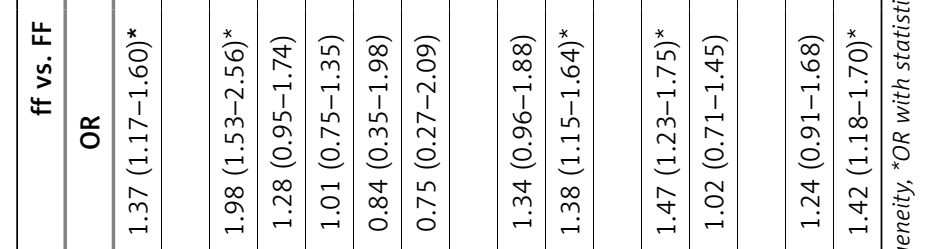

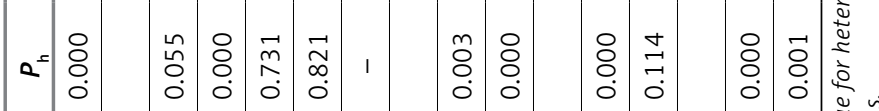

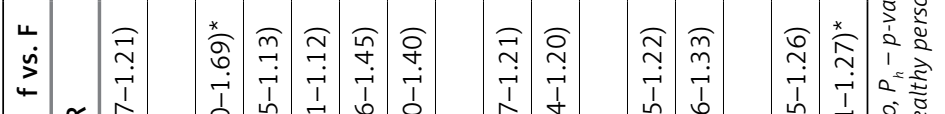

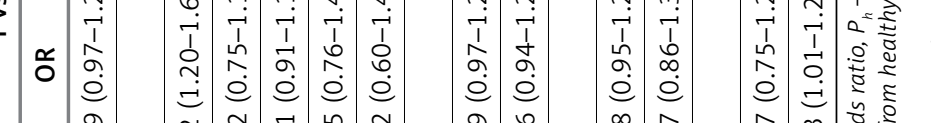

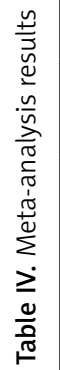

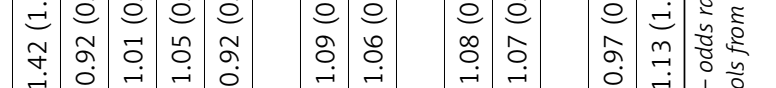

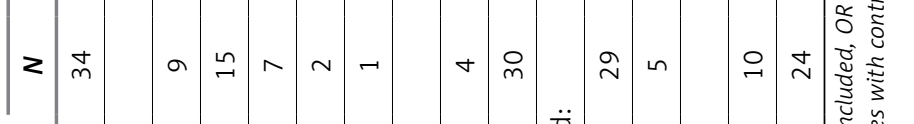
1

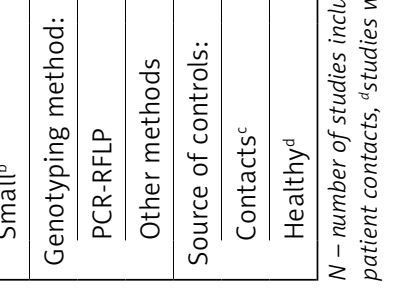

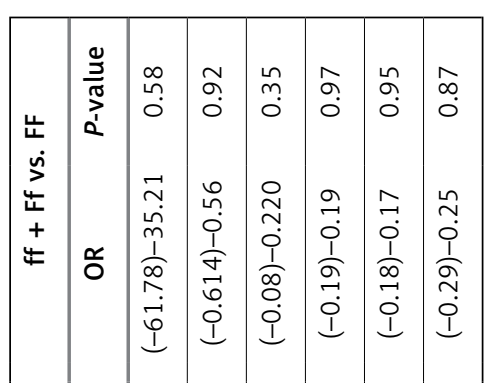

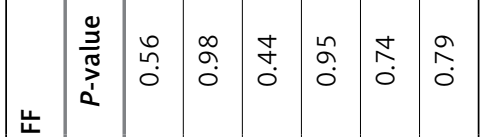

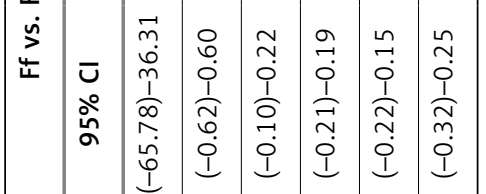

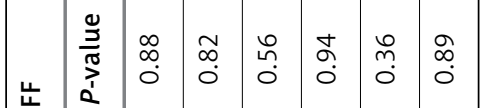

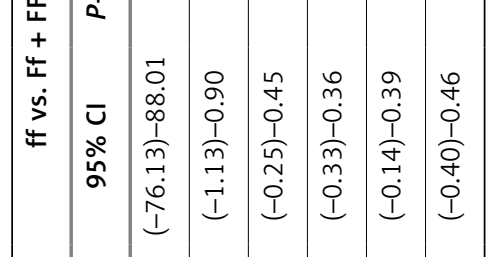

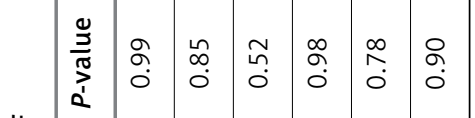

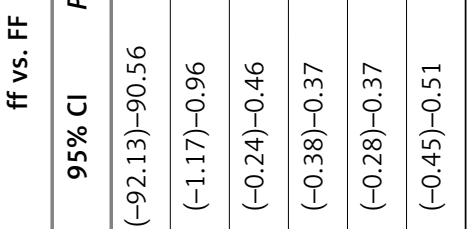

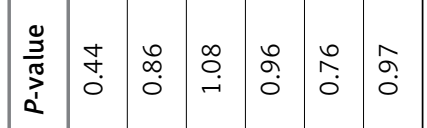

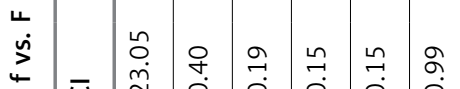

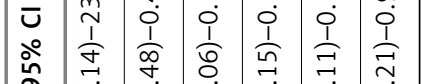

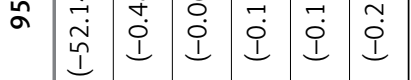
$z \dot{m} \dot{m} \dot{m} \vec{m} \dot{m} \vec{m}$

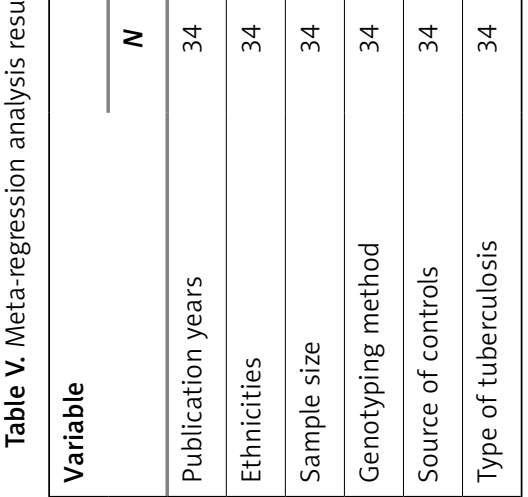


Table VI. Sensitivity analyses of study with controls not in HWE excluded

\begin{tabular}{|lcccc|}
\hline $\begin{array}{l}\text { Study with controls } \\
\text { not in HWE excluded }\end{array}$ & $\begin{array}{c}\text { Summarized } \\
\text { odds ratio }(95 \% \mathrm{Cl})\end{array}$ & No. of included studies & $\boldsymbol{I}^{2}(\%)$ & $P$-value \\
\hline f vs. F & $1.097(0.978-1.229)$ & 31 & 65.3 & 0.113 \\
\hline ff vs. FF & $1.323(1.037-1.689)$ & 31 & 52.3 & 0.025 \\
\hline ff vs. Ff + FF & $1.320(1.083-1.608)$ & 31 & 39.7 & 0.006 \\
\hline Ff vs. FF & $1.042(0.917-1.185)$ & 31 & 47.4 & 0.526 \\
\hline ff + Ff vs. FF & $1.085(0.945-1.246)$ & 31 & 58.8 & 0.246 \\
\hline
\end{tabular}

$\mathrm{Cl}$ - confidence interval.

\section{References}

1. World Health Organization. Global tuberculosis Report 2014. Geneva: World Health Organization.

2. Rahman S, Gudetta B, Fink J, et al. Compartmentalization of immune responses in human tuberculosis: few CD8+ effector T cells but elevated levels of FoxP3+ regulatory $T$ cells in the granulomatous lesions. Am J Pathol 2009; 174: 2211-24.

3. Bowen EF, Rice PS, Cooke NT, Whitfield RJ, Rayner CF. HIV seroprevalence by anonymous testing in patients with Mycobacterium tuberculosis and in tuberculosis contacts. Lancet 2000; 356: 1488-9.

4. Hillerdal G. Environmental dangers: asbestos and tuberculosis. Respiration 2000; 67: 134.

5. Newport MJ, Nejentsev S. Genetics of susceptibility to tuberculosis in humans. Monaldi Arch Chest Dis 2004; 61: 102-11.

6. Borgdorff MW. The NRAMP1 gene and susceptibility to tuberculosis. N Engl J Med 1998; 339: 199-200; author reply 200-201.

7. Amirzargar AA, Yalda A, Hajabolbaghi M, Khosravi F, Jabbari H. The association of HLA-DRB, DQA1, DQB1 alleles and haplotype frequency in Iranian patients with pulmonary tuberculosis. Int J Tuberc Lung Dis 2004; 8: 1017-21.

8. Selvaraj P, Chandra G, Kurian SM, et al. Association of vitamin D receptor gene variants of Bsml, Apal and Fokl polymorphisms with susceptibility or resistance to pulmonary tuberculosis. Curr Sci 2003; 84: 1564-8.

9. Ralph AP, Lucas RM. Vitamin D and tuberculosis: hope or hype? Med J Aust 2013; 199: 648-9.

10. Wilkinson RJ, Llewelyn M, Toossi Z, et al. Influence of vitamin $\mathrm{D}$ deficiency and vitamin $\mathrm{D}$ receptor polymorphisms on tuberculosis among Gujarati Asians in west London: a case-control study. Lancet 2000; 355: 618-21.

11. Joshi L, Ponnana M, Penmetsa SR, et al. Serum vitamin D levels and VDR polymorphisms (Bsml and Fokl) in patients and their household contacts susceptible to tuberculosis. Scand I Immunol 2014; 79: 113-9.

12. Wu F, Zhang W, Zhang L, et al. NRAMP1, VDR, HLA-DRB1, and HLA-DQB1 gene polymorphisms in susceptibility to tuberculosis among the Chinese Kazakh population: a case-control study. Biomed Res Int 2013; 2013: 484535.

13. Sinaga BYM, Amin M, Siregar Y, Sarumpaet SM. Correlation between vitamin D receptor gene FOKI and BSMI polymorphisms and the susceptibility to pulmonary tuberculosis in an Indonesian Batak-ethnic population. Acta Med Indones 2014; 46: 275-82.

14. Arji N, Busson M, Iraqi G, et al. Genetic diversity of TLR2, TLR4, and VDR loci and pulmonary tuberculosis in Moroccan patients. J Infect Dev Ctries 2014; 8: 430-40.

15. Aida $M$, Abdellah A. Vitamin $D$ receptor gene polymorphism and 25 hydroxy vitamin D levels in Egyptian pa- tients with pulmonary tuberculosis. Egypt J Chest Dis Tubercul 2014; 63: 651-5.

16. Yiallourou Al, Ekonomou E, Tsamadias V, et al. Association of Fokl and Pvull polymorphisms with breast cancer staging and survival among Caucasian women: a prospective study. J BUON 2014; 19: 633-42.

17. Chen C, Liu Q, Zhu L, Yang H, Lu W. Vitamin D receptor gene polymorphisms on the risk of tuberculosis, a meta-analysis of 29 case-control studies. PLoS One 2013; 8: e83843.

18. Gao L, Tao Y, Zhang L, Jin Q. Vitamin D receptor genetic polymorphisms and tuberculosis: updated systematic review and meta-analysis. Int I Tuberc Lung Dis 2010; 14: 15-23.

19. Kim J, Ahn JH, Park CK, et al. Influence of vitamin D receptor polymorphism on tuberculosis among South Korean. Chest 2011; 140: 780A.

20. Kang TJ, Jin SH, Yeum CE, et al. Vitamin D receptor gene Taq I, Bsm I and Fok I polymorphisms in Korean patients with tuberculosis. Immune Network 2011; 11: 253-7.

21. Liu YD, Xiao HP, Sha W, et al. Distribution of vitamin D receptor gene polymorphisms among new and recurrent pulmonary tuberculosis. Chin J Infect Chemother 2008; 8: 289-92.

22. Chen $X R$, Feng $Y L$, Ma $Y$, et al. Study on the association of two polymorphisms of the vitamin $\mathrm{D}$ receptor (VDR) gene with the susceptibility to pulmonary tuberculosis (PTB) in Chinese Tibetans. J Si Chuan Univ (Med Sci Edi) 2006; 37: 847-51.

23. Selvaraj P, Kurian SM, Chandra G, et al. Vitamin D receptor gene variants of Bsml, Apal, Taql, and Fokl polymorphisms in spinal tuberculosis. Clin Genet 2004; 65: 73-6.

24. Liu W, Zhang CY, Wu XM, et al. A case - control study on the vitamin $D$ receptor gene polymorphisms and susceptibility to pulmonary tuberculosis. Chin J Epidemiol 2004; 24: 389-92.

25. Marashian SM, Farnia P, Seyf S, Anoosheh S, Velayati AA. Evaluating the role of vitamin D receptor polymorphisms on susceptibility to tuberculosis among Iranian patients: a case-control study. Tuberk Toraks 2010; 58: $147-53$.

26. Rathored J, Sharma SK, Singh B, et al. Risk and outcome of multidrug-resistant tuberculosis: vitamin $D$ receptor polymorphisms and serum 25(OH)D. Int J Tuberc Lung Dis 2012; 16: 1522-8.

27. Singh A, Gaughan JP, Kashyap VK. SLC11A1 and VDR gene variants and susceptibility to tuberculosis and disease progression in East India. Int J Tuberc Lung Dis 2011; 15: 1468-74.

28. Sharma PR, Singh S, Jena M, et al. Coding and non-coding polymorphisms in VDR gene and susceptibility to pulmonary tuberculosis in tribes, castes and Muslims of Central India. Infect Genet Evol 2011; 11: 1456-61. 
29. Zhang HO, Deng A, Guo CF, et al. Association between fold polymorphism in vitamin $D$ receptor gene and susceptibility to spinal tuberculosis in Chinese Han population. Arch Med Res 2010; 41: 46-9.

30. Banoei MM, Mirsaeidi MS, Houshmand M, et al. Vitamin $D$ receptor homozygote mutant $t \mathrm{t}$ and $\mathrm{bb}$ are associated with susceptibility to pulmonary tuberculosis in the Iranian population. Int J Infect Dis 2010; 14: E84-5.

31. Merza M, Farnia P, Anoosheh S, et al. The NRAMPI, VDR and TNF-alpha gene polymorphisms in Iranian tuberculosis patients: the study on host susceptibility. Braz J Infect Dis 2009; 13: 252-6.

32. Vidyarani M, Selvaraj P, Raghavan S, Narayanan PR. Regulatory role of 1, 25-dihydroxyvitamin D3 and vitamin $D$ receptor gene variants on intracellular granzyme A expression in pulmonary tuberculosis. Exp Mol Pathol 2009; 86: 69-73.

33. Selvaraj P, Prabhu Anand S, Harishankar M, Alagarasu K. Plasma 1,25 dihydroxy vitamin D3 level and expression of vitamin $\mathrm{D}$ receptor and cathelicidin in pulmonary tuberculosis. J Clin Immunol 2009; 29: 470-8.

34. Alagarasu K, Selvaraj P, Swaminathan S, Narendran G Narayanan PR. 5' Regulatory and 3' untranslated region polymorphisms of vitamin D receptor gene in South Indian HIV and HIV-TB patients. J Clin Immunol 2009; 29: 196-204.

35. Selvaraj P, Vidyarani M, Alagarasu K, Prabhu Anand S, Narayanan PR. Regulatory role of promoter and 3' UTR variants of vitamin $D$ receptor gene on cytokine response in pulmonary tuberculosis. J Clin Immunol 2008; 28: 306-13.

36. Selvaraj P, Chandra G, Jawahar MS, et al. Regulatory role of vitamin D receptor gene variants of Bsm I, Apa I, Taq I, and Fok I polymorphisms on macrophage phagocytosis and lymphoproliferative response to mycobacterium tuberculosis antigen in pulmonary tuberculosis. J Clin Immunol 2004; 24: 523-32.

37. Liu W, Cao WC, Zhang CY, et al. VDR and NRAMP1 gene polymorphisms in susceptibility to pulmonary tuberculosis among the Chinese Han population: a case-control study. Int J Tuberc Lung Dis 2004; 8: 428-34.

38. Olesen R, Wejse C, Velez DR, et al. DC-SIGN (CD209), pentraxin 3 and vitamin $D$ receptor gene variants associate with pulmonary tuberculosis risk in West Africans. Genes Immun 2007; 8: 456-67.

39. Babb C, van der Merwe L, Beyers N, et al. Vitamin D receptor gene polymorphisms and sputum conversion time in pulmonary tuberculosis patients. Tuberculosis (Edinb) 2007; 87: 295-302.

40. Søborg C, Andersen AB, Range N, et al. Influence of candidate susceptibility genes on tuberculosis in a high endemic region. Mol Immunol 2007; 44: 2213-20.

41. Lombard Z, Dalton DL, Venter PA, Williams RC, Bornman $L$. Association of HLA-DR, -DQ, and vitamin $D$ receptor alleles and haplotypes with tuberculosis in the Venda of South Africa. Hum Immunol 2006; 67: 643-54.

42. Bornman L, Campbell SJ, Fielding K, et al. Vitamin D receptor polymorphisms and susceptibility to tuberculosis in West Africa: a case-control and family study. J Infect Dis 2004; 190: 1631-41.

43. Ates O, Dolek B, Dalyan L, et al. The association between Bsml variant of vitamin D receptor gene and susceptibility to tuberculosis. Mol Biol Rep 2011; 38: 2633-6.

44. Wilbur AK, Kubatko LS, Hurtado AM, Hill KR, Stone AC. Vitamin D receptor gene polymorphisms and susceptibility $M$. tuberculosis in native Paraguayans. Tuberculosis (Edinb) 2007; 87: 329-37.
45. Roth DE, Soto G, Arenas F, et al. Association between vitamin $\mathrm{D}$ receptor gene polymorphisms and response to treatment of pulmonary tuberculosis. J Infect Dis 2004; 190: 920-7.

46. Li L, Wu B, Liu JY, Yang LB. Vitamin D receptor gene poly morphisms and type 2 diabetes: a meta-analysis. Arch Med Res 2013; 44: 235-41. 